This figure "kirkpatrick.fig1a.jpg" is available in "jpg" format from: http://arxiv.org/ps/astro-ph/0003317v1 
This figure "kirkpatrick.fig1b.jpg" is available in "jpg" format from: http://arxiv.org/ps/astro-ph/0003317v1 
This figure "kirkpatrick.fig1c.jpg" is available in "jpg" format from: http://arxiv.org/ps/astro-ph/0003317v1 
This figure "kirkpatrick.fig1d.jpg" is available in "jpg" format from: http://arxiv.org/ps/astro-ph/0003317v1 
This figure "kirkpatrick.fig1e.jpg" is available in "jpg" format from: http://arxiv.org/ps/astro-ph/0003317v1 
This figure "kirkpatrick.fig1f.jpg" is available in "jpg" format from: http://arxiv.org/ps/astro-ph/0003317v1 
This figure "kirkpatrick.fig1g.jpg" is available in "jpg" format from: http://arxiv.org/ps/astro-ph/0003317v1 
This figure "kirkpatrick.fig2a.jpg" is available in "jpg" format from: http://arxiv.org/ps/astro-ph/0003317v1 
This figure "kirkpatrick.fig2b.jpg" is available in "jpg" format from: http://arxiv.org/ps/astro-ph/0003317v1 
This figure "kirkpatrick.fig2c.jpg" is available in "jpg" format from: http://arxiv.org/ps/astro-ph/0003317v1 
To appear in the Astronomical Journal, July 2000 issue

\title{
Sixty-seven Additional L Dwarfs Discovered by the Two Micron All Sky Survey (2MASS) 円
}

\author{
J. Davy Kirkpatrick ${ }^{2}$, I. Neill Reid ${ }^{3}$, James Liebert ${ }^{4}$, John E. Gizis ${ }^{2}$, Adam J. Burgasser ${ }^{5}$, David \\ G. Monet ${ }^{6}$, Conard C. Dahn ${ }^{6}$, Brant Nelson ${ }^{2}$, and Rik J. Williams ${ }^{7}$
}

\begin{abstract}
We present $J H K_{s}$ photometry, far red spectra, and spectral classifications for an additional $67 \mathrm{~L}$ dwarfs discovered by the Two Micron All Sky Survey. One of the goals of this new search was to locate more examples of the latest L dwarfs. Of the 67 new discoveries, 17 have types of L6 or later. Analysis of these new discoveries shows that $\mathrm{H} \alpha$ emission has yet to be convincingly detected in any $\mathrm{L}$ dwarf later than type L4.5, indicating a decline or absence of chromospheric activity in the latest L dwarfs. Further analysis shows that 16 (and possibly 4 more) of the new L dwarfs are lithium brown dwarfs and that the average line strength for those $\mathrm{L}$ dwarfs showing lithium increases until type $\sim \mathrm{L} 6.5 \mathrm{~V}$ then declines for later types. This disappearance may be the first sign of depletion of atomic lithium as it begins to form into lithium-bearing molecules. Another goal of the search was to locate nearer, brighter L dwarfs of all subtypes. Using absolute magnitudes for $17 \mathrm{~L}$ dwarf systems with trigonometric parallax measurements, we develop spectrophotometric relations to estimate distances to the other L dwarfs. Of the 67 new discoveries, 21 have photometric distances placing them within 25 parsecs of the Sun. A table of all known L and T dwarfs believed to lie within 25 parsecs - 53 in total - is also presented. Using the distance measurement
\end{abstract}

\footnotetext{
${ }^{1}$ Portions of the data presented herein were obtained at the W.M. Keck Observatory which is operated as a scientific partnership among the California Institute of Technology, the University of California, and the National Aeronautics and Space Administration. The Observatory was made possible by the generous financial support of the W.M. Keck Foundation.

${ }^{2}$ Infrared Processing and Analysis Center, MS 100-22, California Institute of Technology, Pasadena, CA 91125; davy@ipac.caltech.edu, gizis@ipac.caltech.edu, nelson@ipac.caltech.edu

${ }^{3}$ Department of Physics and Astronomy, University of Pennsylvania, Philadelphia, PA 19104-6396; inr@herschel.physics.upenn.edu

${ }^{4}$ Steward Observatory, University of Arizona, Tucson, AZ 85721; liebert@as.arizona.edu

${ }^{5}$ Department of Physics, MS 103-33, California Institute of Technology, Pasadena, CA 91125; diver@its.caltech.edu

${ }^{6}$ U.S. Naval Observatory, P.O. Box 1149, Flagstaff, AZ 86002; dgm@nofs.navy.mil, dahn@nofs.navy.mil

${ }^{7}$ Department of Astronomy, MSC 152, California Institute of Technology, Pasadena, CA 91126-0152
} 
of the coolest L dwarf known, we calculate that the gap in temperature between L8 and the warmest known $\mathrm{T}$ dwarfs is less than $350 \mathrm{~K}$ and probably much less. If the transition region between the two classes spans a very small temperature interval, this would explain why no transition objects have yet been uncovered. This evidence, combined with model fits to low-resolution spectra of late-M and early-L dwarfs, indicates that L-class objects span the range $1300 \mathrm{~K} \lesssim T_{\text {eff }} \lesssim 2000 \mathrm{~K}$. The near-infrared color-color diagram shows that L dwarfs fall along a natural, redder extension of the well known $\mathrm{M}$ dwarf track. These near-infrared colors get progressively redder for later spectral types, with the $\mathrm{L}$ dwarf sequence abruptly ending near $\left(J-H, H-K_{s}\right.$, $\left.J-K_{s}\right) \approx(1.3,0.8,2.1)$.

Subject headings: stars: low-mass, brown dwarfs — stars: fundamental parameters infrared: stars — stars: atmospheres — stars: distances

\section{Introduction}

In 1993, the first spectrum of what would later be known as an L dwarf was published (Kirkpatrick, Henry, \& Liebert 1993). This object, GD 165B, had been discovered earlier by Becklin \& Zuckerman (1988) as a resolved companion to a nearby white dwarf. For several years GD 165B remained in a class by itself. Then, beginning in 1997, an explosion of discoveries proved that L dwarfs are quite common in the solar neighborhood (Reid et al. 1999; see also Delfosse et al. 1997; Ruiz, Leggett, \& Allard 1997; Rebolo et al. 1998; Kirkpatrick et al. 1999; Goldman et al. 1999; Martín et al. 1999b; Fan et al. 2000).

Providing a historical parallel to the $\mathrm{L}$ dwarfs are the $\mathrm{T}$ dwarfs, even cooler objects spectroscopically defined as those showing methane at $K$-band (Kirkpatrick et al. 1999; hereafter referred to as Paper I). In 1995, the first spectrum of a $\mathrm{T}$ dwarf was published (Oppenheimer et al. 1995). This object, Gl 229B, had been discovered as a companion to a nearby M dwarf by Nakajima et al. (1995) and remained in a class by itself for several years. Then beginning in 1999, an explosion of discoveries proved that observable $\mathrm{T}$ dwarfs have a space density comparable to that of L dwarfs (Strauss et al. 1999, Burgasser et al. 1999, Cuby et al. 1999, Burgasser et al. 2000a, Tsvetanov et al. 2000, Burgasser et al. 2000c).

Despite the implied space density and the subsequent profusion of cooler, $\mathrm{T}$ dwarf discoveries, the number of known L dwarfs is still small. Additional examples, including a larger number of late-L dwarfs and nearer (brighter) examples of all L subtypes, are needed for further studies including parallax measurement, luminosity and temperature determination, kinematics, binarity, and detailed spectroscopic analyses related to magnetic activity, lithium frequency, atmospheric abundances, dust formation, etc. To this end, we present here another $67 \mathrm{~L}$ dwarfs found during follow-up of candidates selected from Two Micron All Sky Survey (2MASS) data. 


\section{Target Selection and Spectroscopic Confirmation}

In Paper I we searched for objects in the 2MASS data having $J-K_{s} \geq 1.30, K_{s} \leq 14.50$, and no optical counterpart. This technique proved efficient in finding $\mathrm{L}$ dwarfs but was most sensitive

to the earliest types since such a magnitude-limited search samples a much larger volume of space for early-type objects of higher luminosity than it does for late-type objects of lower luminosity. As a result, Paper I contained very few late-L dwarfs. Also, because of the small initial survey area (only 371 sq. deg.) very few brighter, closer L dwarfs were identified. In this paper we address both deficiencies.

To find more examples of the latest L types, we have searched for 2MASS objects having $J-K_{s} \geq 1.7, K_{s} \leq 15.0$, and no optical counterpart on the POSS-II plates. To find nearer examples at all $\mathrm{L}$ types, we have also searched for 2MASS objects having $J-K_{s} \geq 1.3$ and $K_{s} \leq 13.0$ and having either no POSS-II counterpart or a counterpart implying colors of $R-K_{s}>6$.

Spectroscopic follow-up of candidates selected with these search criteria have confirmed a few dozen new L dwarfs in the still growing database. These are listed in Table 1 along with another dozen $\mathrm{L}$ dwarfs that met the same color criteria but were fainter at $K_{s}$. A final $\mathrm{L}$ dwarf with bright magnitudes but bluer colors $\left(J-K_{s}=1.25\right)$ is also included. In Table 1, column 1 gives the object name and columns 2-7 give the 2MASS-measured magnitudes and colors.

\subsection{Keck Observations}

Sixty-five of the L dwarfs in Table 1 were confirmed in 1998 August, 1998 December, 1999 March, and 1999 July using the Low Resolution Imaging Spectrograph (LRIS; Oke et al. 1995) at the $10 \mathrm{~m}$ W. M. Keck Observatory on Mauna Kea, Hawaii. A 400 lines/mm grating blazed at 8500 $\AA$ was used with a $1^{\prime \prime}$ slit and $2048 \times 2048$ CCD to produce 9 - $\AA$-resolution spectra covering the range 6300 - $10100 \AA$. The OG570 order-blocking filter was used to eliminate second-order light. The data were reduced and calibrated using standard IRAF routines. A 1-second dark exposure was used to remove the bias, and quartz-lamp flat-field exposures were used to normalize the response of the detector.

The individual stellar spectra were extracted using the "apextract" routine in IRAF, allowing for the slight curvature of a point-source spectrum viewed through the LRIS optics and using a template where necessary. Wavelength calibration was achieved using neon+argon arc lamp exposures taken after each program object. Finally, the spectra were flux-calibrated using observations of standards LTT 9491, Hiltner 600, LTT 1020, and Feige 56 from Hamuy et al. (1994). The data have not been corrected for telluric absorption, so the atmospheric $\mathrm{O}_{2}$ bands at 6867-7000, 7594-7685 $\AA$ and $\mathrm{H}_{2} \mathrm{O}$ bands at 7186-7273, 8161-8282, 8950-9300, 9300-9650 $\AA$ are still present in the spectra. 


\subsection{Palomar Observations}

The other two L dwarfs in Table 1 were confirmed using the Double Spectrograph (Oke \& Gunn 1982) at the 5m Hale Telescope on Palomar Mountain, California. A 2".0 slit and dichroic beam splitter that splits the light near $6800 \AA$ was used. A 316 line $/ \mathrm{mm}$ grating was placed in the red camera for coverage from 6800 to $9150 \AA$ at a resolution of $10 \AA$. A 300 line $/ \mathrm{mm}$ grating was used in the blue camera to cover the range 3375 to $6825 \AA$, but neither of the L dwarfs had flux detected in the blue. Reductions were identical to those described for the Keck data above.

The telescope used for the spectroscopic observation of each target is listed in column 8 of Table 1 along with the observation date in column 9 and exposure time in column 10. Finding charts for each of these L dwarfs are shown in Figure 1.

\section{Spectroscopic Classification}

Spectral types were assigned following the guidelines established in Paper I. The CrH-a, Rb-b/TiO-b, Cs-a/VO-b, and Color-d ratios, as defined in Paper I, were measured from each spectrum. These are tabulated in columns 2-5 of Table 2 where the names of the $\mathrm{L}$ dwarfs are given in column 1. The values in parentheses after the measured value of each ratio are the class or range in class that most closely corresponds to that value, as judged from the primary standards plotted in Figures 10-12 of Paper I. For those spectra whose ratios suggest a type earlier that L5, we have listed in column 6 the spectral class of the primary spectrum that best fits the K I profile. These primary spectra are the ones given in Table 6 of Paper I. The types implied by the three CrH-a, Rb-b/TiO-b, and Cs-a/VO-b ratios along with the type implied by either the Color-d ratio (for types $>$ L5) or K I fit (for types $\leq$ L5) have been medianed to produce the final spectral type.

This procedure works well except for spectra with lower signal-to-noise. In these spectra, the narrower indices ( $\mathrm{Rb}-\mathrm{b} / \mathrm{TiO}-\mathrm{b}$ and $\mathrm{Cs}-\mathrm{a} / \mathrm{VO}-\mathrm{b})$ are more prone to uncertainties due to random noise spikes. For such cases, a by-eye comparison to the alkali and oxide features of the primary standards is a more reliable indicator of type. Specifically, there are twenty-one lower quality spectra in Table 2 where the $\mathrm{Rb}-\mathrm{b} / \mathrm{TiO}-\mathrm{b}$ and $\mathrm{Cs}-\mathrm{a} / \mathrm{VO}-\mathrm{b}$ ratios have been replaced by the best

fit to the 7800-8600 $\AA$ region encompassing the Rb I doublet, the Cs I $8521 \AA$ line, the VO band near $7900 \AA$, and the TiO band at $8432 \AA$. These best fits are listed in column 7 of Table 2 .

Final spectral types for each object are listed in column 8 of Table 2. The 67 new L dwarf spectra are displayed in order of increasing L subtype in Figure 2. Values of the spectral ratios as a function of final spectral class are illustrated in Figure 3 with values for the $25 \mathrm{~L}$ dwarfs from Paper I shown for comparison. 


\section{Spectroscopic Analyses}

The sample in Table 2 not only represents a huge increase in the number of $\mathrm{L}$ dwarfs known in general, but it also includes another 17 dwarfs with types of L6 or later. This larger sample can be used to study trends that evolve with spectral type.

\section{1. $\mathbf{H} \alpha$ Emission}

The absence of $\mathrm{H} \alpha$ emission in late $\mathrm{L}$ dwarfs, as hinted at in Paper I, can now be reinvestigated. Column 9 of Table 2 gives for all 67 spectra the measure of (or upper limit to) the equivalent width of the $\mathrm{H} \alpha$ emission feature. Detailed spectra near $\mathrm{H} \alpha$ are shown in Figure 4 for those objects exhibiting emission or possible emission. Figure 5a shows these equivalent widths as a function of spectral subclass. To provide a larger sample, the $25 \mathrm{~L}$ dwarfs from Paper I have also been included. As the figure shows, typical $\mathrm{H} \alpha$ strengths for those early L dwarfs with emission are generally a few $\AA$. $\mathrm{H} \alpha$ lines of similar strength would be detectable for several of the late-L dwarfs here, but none shows the line. In fact, the latest $\mathrm{L}$ dwarf with detected $\mathrm{H} \alpha$ emission is the L4.5 dwarf 2MASSW J2224438-015852, and here the 1- $\AA$ equivalent width line is observable only because of the spectacular signal-to-noise in this spectrum.

Figure $5 \mathrm{~b}$ shows as a function of $\mathrm{L}$ subclass the percentage of $\mathrm{L}$ dwarfs having $\mathrm{H} \alpha$ emission. Only those spectra with sufficient signal-to-noise to detect a line of $2 \AA$ equivalent width are included in the computation. For type L0, 60\% show $\mathrm{H} \alpha$ emission of this strength, but the percentage drops markedly for types L1, L2, and L3. For types L4 and L5, H $\alpha$ emission is not detected at $2 \AA$ equivalent width or greater in any of our sample. For many of the latest L dwarfs (L6-L8) the signal-to-noise is too poor to exclude $\mathrm{H} \alpha$ emission at $2 \AA$ equivalent width because our ability to detect low-level $\mathrm{H} \alpha$ emission is compromised by the paucity of red photons. That having been stated, even for those few L6-L8 dwarfs having sufficient signal-to-noise, $\mathrm{H} \alpha$ emission was also not detected.

It should also be noted that $\mathrm{H} \alpha$ strengths for the entire ensemble of $\mathrm{H} \alpha$-emitting $\mathrm{L}$ dwarfs are smaller than in typical dMe stars. Both this and the absence of measureable $\mathrm{H} \alpha$ in mid- and late-L dwarfs indicate a decline in chromospheric activity throughout the L dwarf sequence. Gizis et al. (2000) have used the available data on $\mathrm{L}$ dwarfs with and without $\mathrm{H} \alpha$ emission to conclude that lack of activity may correlate with youth and substellar nature. Such a correlation would explain why $\mathrm{H} \alpha$ emission is not seen in dwarfs later than L4.5 as this subclass corresponds roughly to the temperature at which stellar interior models predict a substellar fraction of $100 \%$. In other words, a mixture of stars and brown dwarfs is expected at earlier L types (where $\mathrm{H} \alpha$ emitters and non- $\mathrm{H} \alpha$ emitters lie), but later than this (where no $\mathrm{H} \alpha$ emitters are found), all objects are expected to be substellar. The seeming anti-correlation between L dwarfs with lithium absorption and those with $\mathrm{H} \alpha$ emission is more evidence in favor of this conclusion. 


\subsection{Lithium Absorption}

Paper I also suggested that lithium disappears (or is much weaker) in the latest L dwarfs, probably due to the formation of lithium-bearing molecules (Burrows \& Sharp 1999, Lodders 1999). We can also reinvestigate this hypothesis. Column 10 of Table 2 gives for each spectrum the measure of (or upper limit to) the equivalent width of the Li I doublet. Detailed spectra near the lithium doublet are shown in Figure 6 for those objects exhibiting Li absorption or possible absorption. Figure 7a shows these equivalent widths as a function of spectral subclass, and L dwarfs from Paper I have been added to increase the sample size.

For early- and mid-L dwarfs with detected lithium, the equivalent width increases with later subclasses. This is the same behavior seen with the ground-state doublets of the other abundant alkalis $\mathrm{Na}$ I and K I. In these objects the cooler temperatures mean that more and more of the alkali atoms are in their neutral state. This effect, along with the pressure broadening and the increased column density through which the emergent radiation passes (thanks to an atmosphere made more transparent by the removal of overlying $\mathrm{TiO}$ and $\mathrm{VO}$ absorption), creates stronger and stronger absorption lines. The main difference is that for lithium, its lower cosmic abundance precludes the development of giant absorption troughs like those produced by sodium and potassium (Reid et al. 2000).

However, the trend of increasing line strength for lithium, unlike sodium and potassium, reverses at types around L6.5-L7 V. Figure 7b shows the percentage of L dwarfs with detectable lithium as a function of spectral subclass. Only those L dwarfs with sufficient signal to see a line of $4 \AA$ equivalent width are used in the calculation. The percentage of $\mathrm{L}$ dwarfs with strong lithium may drop for types L7 and L8 (although our statistics are still poor), but as shown in Figure $7 \mathrm{c}$, the strength of the line when detected is much weaker than that seen for types L5 and L6. In other words, although lithium is sometimes seen in the latest L dwarfs, its strength is greatly diminished. At face value, this seems to run contrary to the expectation that the latest $\mathrm{L}$ dwarfs should show increased line strengths of ground state Li I due to their cooler temperatures.

We interpret this as evidence for $\mathrm{Li}$ depletion due to the formation of lithium-bearing molecules. At temperatures typical of late-M dwarfs, the primary lithium-bearing gas is Li. At cooler temperatures, however, lithium will begin to form molecules such as $\mathrm{LiOH}$, $\mathrm{LiCl}$, or $\mathrm{LiF}$ depending upon the physics of the gas mixture. At the pressures expected in these objects, Lodders (1999) concludes that $\mathrm{LiCl}$ is the molecule responsible for robbing $\mathrm{Li}$ out of the atmosphere. She also shows that $\mathrm{Li}$ and $\mathrm{LiCl}$ should have equal abundances near $1500-1550 \mathrm{~K}$ with $\mathrm{LiCl}$ being dominant at cooler temperatures. Because dwarfs of type L6.5-L7 show clear signs of Li depletion

in their spectra, we can conclude that such dwarfs have temperatures in the $1500 \mathrm{~K}$ realm. A more robust temperature estimate would require observations of a band of $\mathrm{LiCl}$ so that abundances of $\mathrm{Li}$ and $\mathrm{LiCl}$ could be directly compared. 


\section{Distances}

In addition to increasing the sample size of $\mathrm{L}$ dwarfs particularly at the latest types, another goal of our survey was to find nearer examples of all L types. A few of the brighter objects in Table 2 already have measured trigonometric parallaxes, a couple of which are within $10 \mathrm{pc}$ of the Sun. We can use these parallaxes as well as other parallaxes from Dahn (priv. comm.) and from the literature to estimate distances to the rest. Shown in Figure 8 are plots of absolute $J$ magnitude and absolute $K_{s}$ magnitude as a function of spectral class for late-M, L, and T dwarfs. The objects plotted here are listed in Table 3, where the object name and spectral type are given in columns 1-2, the reference for the trigonometric parallax measure is given in column 3 , and $M_{J}$ and $M_{K s}$ values (derived from 2MASS $J$ and $K_{s}$ photometry) are given in columns 4-5.

A second-order least squares fit to the late-M and L dwarfs gives the following relations between absolute magnitude and spectral class:

$$
\begin{gathered}
M_{J}=11.780+0.198(\text { subclass })+0.023(\text { subclass })^{2} \\
M_{K_{s}}=10.450+0.127(\text { subclass })+0.023(\text { subclass })^{2}
\end{gathered}
$$

where subclass $=-1$ for M9 V, -0.5 for M9.5 V, 0 for L0 V, 0.5 for L0.5 V, etc. These relations are valid between M9 V and L8 V and are plotted as solid lines in Figures 8a and 8b.

With equations (1) and (2) in hand, we can now provide distance estimates to the $62 \mathrm{~L}$ dwarfs lacking trigonometric parallaxes in Table 2. Here we compare the measured $J$ and $K_{s}$ magnitudes to the absolute magnitudes implied by plugging the spectral type into equations (1) and (2). The average of the $J$ and $K_{s}$ distance estimates is listed in column 11 of Table 2 .

Several of these $\mathrm{L}$ dwarfs have measured distances or distance estimates making them eligible for inclusion in the Catalogue of Nearby Stars (Gliese \& Jahreiss 1991); i.e., they lie within 25 pc of the Sun. Table 4 lists from Table 2, from Paper I, and from the literature all known L dwarfs that are within or possibly within this 25-pc limit. Also listed are the known T dwarfs believed to be within 25 parsecs. Table 4 is ordered by spectral type, with the early L dwarfs at the top and $\mathrm{T}$ dwarfs at the bottom. This list represents the nearest, brightest examples of each class and as such is the list of choice for further follow-up studies. Columns 1 and 2 give the object name and discovery paper. Columns 3-5 list the spectral type and $J$ and $K_{s}$ magnitudes. Column 6 gives the estimated distance computed from equation (1), and column 7 gives the estimated distance computed from equation (2) are left blank and the measured distance is instead listed in column 8 .

\footnotetext{
${ }^{8}$ For T dwarfs, distances are estimated using the method of Burgasser et al. (1999)
} 


\section{The Spectroscopic Gap Between L and T Dwarfs}

In Paper I the issue was also raised as to whether the L dwarf sequence extended to types later than L8. We have calculated that our current search for the latest L dwarfs covers approximately $10 \%$ of the sky. Despite this large survey area, however, we still have not uncovered any L dwarf significantly later than the L8 V presented in Paper I, confirming the conclusions of Paper I. Objects cooler than L8 V have been found in the 2MASS data (Burgasser et al. 1999; Burgasser et al. 2000a) but these are $\mathrm{T}$ dwarfs.

Spectroscopically, late-L dwarfs and $\mathrm{T}$ dwarfs are quite different at $J, H$, and $K$ bands, $\mathrm{T}$ dwarfs having strong bands of $\mathrm{CH}_{4}$ that are absent in $\mathrm{L}$ dwarfs. In the far red, on the other hand, $\mathrm{L}$ and $\mathrm{T}$ dwarfs are more similar. In this region, the weak $\mathrm{FeH}$ bands seen in an $\mathrm{L} 8 \mathrm{~V}$ can also be seen in the spectra of some T dwarfs (Burgasser et al. 2000b), and both L and T dwarfs show $\mathrm{H}_{2} \mathrm{O}$, Cs I, and K I absorption (Liebert et al. 2000).

The near-infrared spectral differences suggest that objects intermediate between L8 V and the $\mathrm{T}$ dwarfs will be difficult to distinguish in the 2MASS data because they presumably would have, at the inception of $\mathrm{CH}_{4}$ formation, colors intermediate between those of an L8 $\mathrm{V}\left(J-K_{s} \approx 2.1\right)$ and a typical $\mathrm{T}$ dwarf $\left(J-K_{s} \approx 0.0\right)$. As $\S 8$ below describes more fully, the 2MASS L dwarf discoveries appear to have, within some cosmic scatter, a roughly monotonic relation of $J-K_{s}$ color with spectral type. That is, there is no evidence that the $J-K_{s}$ color turns bluer for the L8 V discoveries, yet no L dwarfs with $J-K_{s}$ colors redder than 2.1 and with types later than L8 have been found despite exhaustive search efforts.

On the other hand, the spectral similarities in the far red portion of $\mathrm{L}$ and $\mathrm{T}$ dwarf spectra mean that colors in that region are not affected by the blue reversal seen in the near-infrared. A search of preliminary data from the Sloan Digital Sky Survey (SDSS) has uncovered 7 L dwarfs (Fan et al. 2000) and $2 \mathrm{~T}$ dwarfs (Strauss et al. 1999; Tsvetanov et al. 2000) based on $i^{*}-z^{*}$ colors, and it appears that the $i^{*}-z^{*}$ color may be monotonic across the L/T border. Specifically, $i^{*}-z^{*}$ increases from 1.8 at L0 V, to 2.3 at L5 V, to 2.6 at L8 V, to $\sim 4$ for the 2 SDSS T dwarfs. (The jump in $i^{*}-z^{*}$ color between the latest $\mathrm{L}$ dwarfs and the $\mathrm{T}$ dwarfs may be caused by the increasing strength of the K I ground-state resonance doublet, which robs the spectrum of much of its $i^{*}$-band flux. This may be the analogue to the $\sim 1$-magnitude jump in $V-I$ color noted by Reid et al. (2000) between L4 V and L5 V, an effect thought to be caused by the broadening of the ground-state Na I doublet.) Selection for objects intermediate between types L and T would thus be unbiased using SDSS colors. Although the statistics is still based on small numbers, no such objects have been uncovered despite a successful search for dwarfs on either side of the apparent gap.

These results can be explained if the transition between dwarfs of type L $8 \mathrm{~V}$ and the known $\mathrm{T}$ dwarfs covers a small range in temperature, implying that such intermediate objects are relatively rare. Further observational evidence supports this theory: The best studied T dwarf, Gl 229B, is known to have a temperature near 950K (Marley et al. 1996, Allard et al. 1996) and absolute 
bolometric magnitude of 17.7 (Matthews et al. 1996, Leggett et al. 1999). Based on spectral appearance, the coolest L dwarf known is probably the L8 dwarf 2MASSW J1523226+301456 (Gl $584 \mathrm{C}$ ). As seen in Table 3, Gl 584C has $M_{J}=15.0$, a mere 0.4 mag brighter than the $M_{J}=15.4$ value for Gl 229B. Based on the Tinney et al. (1993) measurements of $B C_{J}=1.9$ for the M9 dwarf LHS 2924 and $B C_{J}=1.7$ for the L4 dwarf GD 165B, we extrapolate to $B C_{J} \approx 1.3$ for L8 dwarfs like Gl 584C. (See also Reid et al. 1999.) This implies $M_{b o l} \approx 16.3$ for Gl 584C. Both Gl 229B and Gl 584C are brown dwarfs and thus to first order have very similar radii (Kumar 1963). Even to second order, because both objects are thought to have masses near $0.045 M_{\odot}$ and ages older than $\sim 0.5$ Gyr (Kirkpatrick et al. 2000), model calculations show that they should have radii that are very nearly identical (Baraffe \& Chabrier priv. comm., Burrows et al. 1997). From the Stefan-Boltzmann law, we can therefore deduce that the temperature difference between Gl 584C and Gl $229 \mathrm{~B}$ is only $\sim 350 \mathrm{~K}$, thus giving Gl $584 \mathrm{C} T_{\text {eff }} \approx 1300 \mathrm{~K}$.

Because the available evidence suggests that the T dwarf SDSS $1624+0029$ is warmer than Gl 229B (Nakajima et al. 2000; Liebert et al. 2000; Burgasser et al. 2000b), this means that the gap between L8 V and the warmest T dwarfs is less than $350 \mathrm{~K}$. We believe that the temperature range spanned by the gap is considerably less than $350 \mathrm{~K}$ for two reasons: (1) Equilibrium thermochemistry of $\mathrm{CO}$ and $\mathrm{CH}_{4}$ (Lodders 1999, Burrows \& Sharp 1999) would suggest that the warmest $\mathrm{T}$ dwarfs extend up to $1200 \mathrm{~K}$ or above. (2) Our confirmation of 100 2MASS discoveries in the $\sim 700 \mathrm{~K} \mathrm{~L}$ dwarf temperature range would imply a considerable number of objects falling in a transition region as broad as $350 \mathrm{~K}$, since L dwarfs and 2MASS-observable $\mathrm{T}$ dwarfs are known to have similar space densities. Even though the 2MASS color cuts employed here and by the Burgasser search only partly cover the transition region, the number of objects implied by a $350 \mathrm{~K}$ gap would suggest that at least a few of these should have already been detected. All have so far escaped discovery. We conclude, therefore, that the gap must be much smaller than $350 \mathrm{~K}$ and possibly even less than $100 \mathrm{~K}$.

One final argument in favor of a transition region spanning a very small temperature interval can be made using the recent discovery of the bright T dwarf 2MASSW J0559-1404 (Burgasser et al. 2000c). This object has far weaker methane bands than any other $\mathrm{T}$ dwarf, a property that Burgasser et al. ascribe to warmer temperature. This would make 2MASSW J0559-1404 warmer than SDSS 1624+0029, which as noted above is believed to be warmer than $950 \mathrm{~K}$. Despite the weaker methane bands in 2MASSW J0559-1404, however, its near-infrared color of $J-K_{s}=0.22 \pm 0.06$ is comparable to that of other $\mathrm{T}$ dwarfs yet still distinctly bluer, by $\sim 1.8$ magnitudes, than the average $J-K_{s}$ value for an L8 V. Forthcoming parallax and bolometric luminosity measurements for 2MASSW J0559-1404 will allow us to determine an accurate temperature, thus allowing better observational constraints on the temperature range spanned by the $\mathrm{L} / \mathrm{T}$ transition region. 


\section{L Dwarf Temperature Scale}

L0 dwarfs show weaker VO bands than late-M dwarfs presumably because vanadium has begun to condense out of the atmospheres of the L0 dwarfs. Based on the thermochemical equilibrium analysis of Lodders (1999), dwarfs of type L0 must then have temperatures near 2000K where perovskite formation begins to rob the chromosphere of its vanadium. Thermochemical equilibrium calculations by Burrows \& Sharp (1999) also support $T_{\text {eff }} \approx 2000 \mathrm{~K}$ for L0 dwarfs. Fits of low-resolution red/near-infrared spectra to atmospheric models including grain and/or dust opacities give $T_{\text {eff }} \approx 2000-2200 \mathrm{~K}$ for the M9 dwarf LHS 2924 (Jones \& Tsuji 1997; Tsuji, Ohnaka, \& Aoki 1996) and $T_{\text {eff }} \approx 1900$ for the L2 dwarf Kelu-1 (Ruiz et al. 1997), again suggesting that type L0 has $T_{\text {eff }} \approx 2000 \mathrm{~K}$.

Given the temperature derivation of G1 584C from the previous section, we can conclude that the $\mathrm{L}$ dwarf sequence spans a range of effective temperature from $\sim 1300 \mathrm{~K}$ to $\sim 2000 \mathrm{~K}$. This is in good agreement with the scale suggested by Reid et al. (1999) and spans a wider range than the conservative scale proposed in Paper I.

Basri et al. (2000) deduce a temperature range of 1600 to $2200 \mathrm{~K}$ for the $\mathrm{L}$ dwarf sequence by comparing high resolution observations of alkali line profiles to allard's atmospheric models that include dust formation and condensation. Using the dusty atmospheres of Tsuji and of Allard, Pavlenko et al. (2000) show that acceptable fits are provided to the far red spectra of late-M, L, and $\mathrm{T}$ dwarfs if an additional opacity source (molecular/dust absorption or dust scattering) is invoked. These authors derive a temperature scale running from $1200 \pm 200 \mathrm{~K}$ for type L7 V to $2200 \pm 200 \mathrm{~K}$ for M9.5 V. The intercomparison of results shows that the largest disagreement between scales

occurs at the coolest temperatures. Here, more work is needed before model atmospheres produce consistent answers when comparing two different wavelength regimes (like the far red and the near-infrared) or when comparing high-resolution line profile fits to fits of low-resolution spectral energy distributions. Nevertheless, at this early stage in the study of L dwarfs, it is reassuring that different approaches lead to similar conclusions. Independent determinations of effective temperatures through direct measures of luminosities and radii are, however, still badly needed to constrain and to check the models.

\section{Colors}

Armed with a much larger sample of L dwarfs than that presented in Paper I, we can also reinvestigate the color space occupied by $\mathrm{L}$ dwarfs and the trends of color with spectral type. Figure 9 shows the $J-H$ vs. $H-K_{s}$ diagram for $\mathrm{M}$ dwarfs (solid circles), L dwarfs (open circles), and $\mathrm{T}$ dwarfs (open stars). Data for the early-M dwarfs comes from the compilation of Leggett (1992). Colors for late-M dwarfs and L dwarfs come from 2MASS data (Gizis et al. 2000, Paper I, this paper). Colors for T dwarfs are taken from 2MASS (Burgasser et al. 1999, 2000a, 2000c) and from the literature (Matthews et al. 1996, Strauss et al. 1999, Tsvetanov et al. 2000). For L 
dwarfs having 2MASS photometric errors of 0.10 mag or larger in either $J, H$, or $K_{s}$, small open circles are plotted; L dwarfs with more accurate magnitudes are plotted as larger open circles. Superimposed here are the dwarf track (solid line, obscured by the M dwarfs in the middle part of the diagram) and giant track (dashed line) from Bessell \& Brett (1988).

This figure shows that $\mathrm{L}$ dwarfs lie on a red extension of the familiar dwarf track. Those $\mathrm{L}$ dwarfs with well measured colors (larger open circles) fall in an area from, roughly, $\left(J-H, H-K_{s}\right)$ $=(0.8,0.5)$ to $(1.3,0.8)$ and have a cosmic scatter similar to that seen for the M dwarfs. However, all indications are that the track abruptly stops at the red end and that slightly cooler objects are sent - via a track on this diagram yet to be observationally determined - to an area near $\left(J-H, H-K_{s}\right)=(0,0)$ where the $\mathrm{T}$ dwarfs lie. Based on arguments in the previous section, this transition track likely covers a very small range in temperature and thus will contain relatively fewer objects.

Figure 10 shows the average near-infrared colors of late-M through late-L dwarfs. Colors have been averaged into spectral class bins with half subclass spacing. These straight averages (solid circles) are plotted as a function of spectral class in Figure 10 and tabulated in Table 5. The number of objects contributing to each average is listed in the last column of Table 5 . Also plotted in Figure 10 are the resulting weighted averages (open circles) where objects with more accurately measured photometry are given higher weights than those with poorly measured photometry. These weighted averages should be treated with caution however as their resulting errors underestimate the inherent object-to-object scatter in the color measures.

The photometry of Figure 10 and Table 5 is measured by 2MASS and is taken from Gizis et al. (2000), Paper I, and this paper. Colors for types earlier than M8 are not shown because as Gizis et al. (2000) show that their color-based selection of late-M dwarfs is biased at types earlier than this. Two other biases are, on the other hand, still present for this sample of L dwarfs: First, early-L types will be biased due to the $J-K_{s} \geq 1.30$ color criterion employed both in Paper I and this paper. This should tend to inflate the observed colors of the early-L dwarfs relative to a bias-free sample. This effect will be partly mitigated by the inclusion of L dwarfs from Gizis et al. (2000) since a more relaxed color cut was used there, but the number of L dwarfs in their sample is regrettably small. Second, the additional $J-K_{s} \geq 1.70$ color constraint employed in this paper will tend to inflate the colors of mid- to late-L dwarfs.

Despite these biases, several conclusions can still be made based on Figure 10:

(1) Within the scatter of the points, $J-K_{s}$ color increases monotonically from late-M through late-L spectral types and has a maximum of $J-K_{s} \approx 2.1$ for the late-L dwarfs. Even though the $J-K_{s}$ color appears to level off near 2.0 at types of $\sim$ L5 and later, the $J-K_{s} \geq 1.70$ bias discussed above may have artifically inflated the colors of the mid-L dwarfs relative to late- $\mathrm{L}$ dwarfs. The structure seen at early-L types may also be an artifact of the $J-K_{s} \geq 1.30$ bias having inflated the colors of L0 and L1 dwarfs and the L0.5 bin having been based on only 3 objects. 
(2) Given the same arguments above, it also appears that the $J-H$ color increases roughly monotonically with spectral type for L dwarfs.

(3) $H-K_{s}$ color also appears to increase roughly monotonically with increasing spectral type, at least through mid-L. At late-L types, though, the $H-K_{s}$ color may turn slightly bluer. The $H-K_{s}$ color, however, covers a smaller range than the other two colors, and the dispersions are also quite large. If there is a blueward dip at the latest L types, it may mean that the pressure-induced $\mathrm{H}_{2}$ opacity has increased markedly at $K_{s}$ band. Tokunaga \& Kobayashi (1999) overplot the L2 dwarf Kelu-1 with the L4 dwarf GD 165B and the L7 dwarf DENIS-P J0205.4-1159AB and show that the late-L object has a flux deficit at $K$-band relative to the other two L dwarfs, a deficit which they ascribe to increased collision-induced absorption by molecular hydrogen. This possible blueward dip of $H-K_{s}$ color for the late-L dwarfs and (if verified) its cause need to be studied further with improved photometry and near-infrared spectroscopic follow-up.

Figure 8 has shown that absolute $J$ and $K_{s}$ magnitudes are well correlated with L dwarf spectral subclass. As shown in Figure 11, however, the correlation of $M_{J}$ and $M_{K s}$ with individual $J-K_{s}$ colors exhibits much larger scatter than the correlation of $M_{J}$ and $M_{K s}$ with spectral type. In other words, $\mathrm{L}$ dwarf distance estimates derived from $J-K_{s}$ colors have much larger uncertainties than those derived from spectral type. Part of this scatter is simply due to the uncertainties in 2MASS photometry, typically $\pm 0.07 \mathrm{mag}$ for $J-K_{s}$ though occasionally larger. More importantly, perhaps, this figure demonstrates the intrinsic limitation of estimating spectral types and photometric distances using colors of small baseline. An additional, possible reason for the larger scatter in Figure 11b and 11d is that our spectral type is derived from far red spectra,

and here dust may be playing a minimal role in shaping the spectrum. In the near-infrared, on the other hand, the presence of dust can lead to a backwarming of the atmosphere, and this will alter the amount of $\mathrm{H}_{2} \mathrm{O}$ and $\mathrm{H}_{2}$ in the photosphere. Because both of these molecules play a critical role in shaping the near-infrared spectrum, slight object-to-object variations in their opacities can lead to differences in near-infrared colors (Chabrier et al. 2000).

\section{Conclusions}

We present spectra for another $67 \mathrm{~L}$ dwarfs discovered during follow-up of sources identified by 2MASS. These together with L dwarfs from Paper I, from Gizis et al. (2000), and from other surveys such as SDSS and DENIS, bring the total of known L dwarfs to well over 100. This sample can be used for a variety of follow-up investigations. The presence of $\mathrm{H} \alpha$ emission is seen to decline rapidly from early- to mid-L dwarfs, and $\mathrm{H} \alpha$ emission is not seen in any L dwarf later than type L4.5. If the lack of $\mathrm{H} \alpha$ emission is an indicator of youth and/or substellarity as Gizis et al. (2000) suggest, then the fraction of $\mathrm{H} \alpha$-emitters to non-emitters at any given L subtype may reflect the fraction of stars to brown dwarfs at that class. Lithium absorption, when detected, is seen to increase in strength from early- to mid-L types, but then declines markedly after 
type L6.5 V. This turnover in lithium strength may herald the depletion of atomic lithium into lithium-bearing molecules and as such would provide a vital clue to the temperature scale for L dwarfs because these reactions are expected roughly around $1500 \mathrm{~K}$. The difference in temperature between the latest L dwarf and Gl 229B is calculated at $\sim 350 \mathrm{~K}$. This means that the gap in temperature between L8 and the warmest of the known $\mathrm{T}$ dwarfs must be significantly less that $350 \mathrm{~K}$ as several of the known T dwarfs are suspected of being warmer than Gl 229B itself. This also means that L dwarfs span the likely temperature range $1300 \mathrm{~K} \lesssim T_{\text {eff }} \lesssim 2000 \mathrm{~K}$. The locus of L dwarfs in near-infrared color space is also shown, and distances estimates are made for all $\mathrm{L}$ and $\mathrm{T}$ dwarfs lacking trigonometric parallax measurements. Even at this early stage in our investigations, researchers have identified $53 \mathrm{~L}$ and $\mathrm{T}$ dwarfs known (or suspected) to be within 25 parsecs of the Sun, clearly indicating that this previously hidden population of cool objects is very large.

JDK, INR, and JL acknowledge funding through a NASA/JPL grant to 2MASS Core Project science. AJB acknowledges support from this grant. JDK, JEG, AJB, BN, and RJW acknowledge the support of the Jet Propulsion Laboratory, California Institute of Technology, which is operated under contract with the National Aeronautics and Space Administration. The finder charts of Figure 1 make use of the Digitized Sky Survey (DSS), which was produced at the Space Telescope Science Institute under U.S. Government grant NAGW-2166. The DSS itself is made possible by the existence of the POSS-I, POSS-II, and UK Schmidt photographic surveys. The Second Palomar Sky Survey (POSS-II) was funded by the Eastman Kodak Company, the National Geographic Society, the Samuel Oschin Foundation, the Alfred Sloan Foundation, the National Science Foundation grants AST 84-08225, AST 87-19465, AST 90-23115, and AST 93-18984, and the National Aeronautics and Space Administration grants NGL 05002140 and NAGW 1710. The UK Schmidt survey was carried out at the UK Schmidt Telescope operated by the Royal Greenwich Observatory Edinburgh with funding from the UK Science and Engineering Research Council, until 1988 June, and thereafter by the Anglo-Australian Observatory. This research has also made use of the SIMBAD database, operated at CDS, Strasbourg, France. JDK would like to thank the rest of the 2MASS team, without whose hard work and dedication this research would not have been possible: Ron Beck, Tom Chester, Roc Cutri, Diane Engler, Tracey Evans, John Fowler, Linda Fullmer, Eric Howard, Robert Hurt, Helene Hyunh, Tom Jarrett, Gene Kopan, Bob Light, Ken Marsh, Howard McCallon, Jeonghee Rho, Mike Skrutskie, Rae Stiening, Raymond Tam, Schuyler Van Dyk, Bill Wheaton, Sherry Wheelock, John White, Cong Xu, and anyone else he may have inadvertently omitted. JDK would also like to thank assistance at Keck by Joel Aycock, Tom Bida, Randy Campbell, Teresa Chelminiak, Gary Puniwai, Ron Quick, Barbara Schaefer, Chuck Sorenson, David Sprayberry, Terry Stickel, Wayne Wack, and Greg Wirth and at Palomar by Rick Burruss, Karl Dunscombe, and Skip Staples. This publication makes use of data from the Two Micron All Sky Survey, which is a joint project of the University of Massachusetts and the Infrared Processing and Analysis Center, funded by the National Aeronautics and Space Administration and the National Science Foundation. 


\section{REFERENCES}

Allard, F., Hauschildt, P. H., Baraffe, I, \& Chabrier, G. 1996, ApJ, 465, L123.

Basri, G., Mohanty, S., Allard, F., Hauschildt, P. H., Delfosse, X., Martín, E. L., Forveille, T. \& Goldman, B. 2000, ApJ, submitted.

Becklin, E. E., \& Zuckerman, B., 1988, Nature, 336, 656.

Bessell, M. S., \& Brett, J. M. 1988, PASP, 100, 1134.

Burgasser, A. J., Kirkpatrick, J. D., Brown, M. E., Reid, I. N., Gizis, J. E., Dahn, C. C., Monet, D. G., Beichman, C. A., Liebert, J., Cutri, R. M., \& Skrutskie, M. F. 1999, ApJL, 522, L65.

Burgasser, A. J., et al. 2000a, ApJ, 531, L57.

Burgasser, A. J., Kirkpatrick, J. D., Reid, I. N., Liebert, J., Brown, M. E., \& Gizis, J. E. 2000b, AJ, submitted.

Burgasser, A. J., et al. 2000c, in prep.

Burrows, A., \& Sharp, C. M., 1999, ApJ, 512, 843.

Burrows, A., et al. 1997, ApJ, 491, 856.

Chabrier, G., Baraffe, I., Allard, F., \& Hauschildt, P. 2000, ApJ, submitted.

Cuby, J. G., Saracco, P., Moorwood, A. F. M., D’Odorico, S., Lidman, C., Comerón, F., \& Spyromilio, J. 1999, A\&A, 349, L41.

Delfosse, X., et al. 1997, A\&A, 327, L25.

Fan, X., et al. 2000, AJ, 119, 928.

Gizis, J. E., Monet, D. G., Reid, I. N., Kirkpatrick, J. D., Liebert, J., \& Williams, R. J. 2000, AJ, submitted.

Gliese, W., \& Jahreiss, H. 1991, Preliminary Version of the Third Catalogue of Nearby Stars, ADC CD-ROM, Vol. 1, No. 1.

Goldman, B. et al. 1999, A\&A, 351, L5.

Hamuy, M., Suntzeff, N. B., Heathcote, S. R., Walker, A. R., Gigoux, P., \& Phillips, M. M. 1994, PASP, 106, 566.

Jones, H. R. A., \& Tsuji, T., 1997, ApJ, 480, L39.

Kirkpatrick, J. D., Burgasser, A. J., Gizis, J. E., Reid, I. N., Dahn, C. C., \& Monet, D. G. 2000, in prep. 
Kirkpatrick, J. D., Henry, T. J., \& Liebert, J. 1993, ApJ, 406, 701.

Kirkpatrick, J. D., Henry, T. J., \& Simons, D. A. 1995, AJ, 109, 797.

Kirkpatrick, J. D., Reid, I. N., Liebert, J., Cutri, R. M., Nelson, B., Beichman, C. A., Dahn, C. C., Monet, D. G., Gizis, J. E., \& Skrutskie, M. F., 1999, ApJ, 519, 802 (Paper I).

Koerner, D. W., Kirkpatrick, J. D., McElwain, M. W., \& Bonaventura, N. R., 1999, ApJL. 526, 25.

Kumar, S. S. 1963, ApJ, 137, 1121.

Leggett, S. K. 1992, ApJS, 82, 351.

Leggett, S. K., Toomey, D. W., Geballe, T. R., \& Brown, R. H. 1999, ApJ, 517, L139.

Liebert, J., Burgasser, A. J., Kirkpatrick, J. D., Reid, I. N., Gizis, J. E., 2000, ApJL, submitted.

Lodders, K., 1999, ApJ, 519, 793.

Marley, M. S., Saumon, D., Guillot, T., Freedman, R. S., Hubbard, W. B., Burrows, A., Lunine, J. I., 1996, Science, 272, 1919.

Martín, E. L., Brandner, W., \& Basri, G. 1999a, Science, 283, 1718.

Martín, E., Delfosse, X., Basri, G., Goldman, B., Forveille, T., \& Zapatero Osorio, M. R., 1999b, AJ, 118, 2466.

Matthews, K., Nakajima, T., Kulkarni, S. R., \& Oppenheimer, B. R., 1996, AJ, 112, 1678.

Monet, D. G., Dahn, C. C., Vrba, F. J., Harris, H. C., Pier, J. R., Luginbuhl, C. B., \& Ables, H. D. 1992, AJ, 103, 638.

Nakajima, T., Oppenheimer, B. R., Kulkarni, S. R., Golimowski, D. A., Matthews, K., \& Durrance, S. T. 1995, Nature, 378, 463.

Nakajima, T., et al. 2000, PASJ, in press.

Oke, J. B., et al. 1995, PASP, 107, 375.

Oke, J. B., \& Gunn, J. E. 1982, PASP, 94, 586.

Oppenheimer, B. R., Kulkarni, S. R., Matthews, K., Nakajima, T., 1995, Science, 270, 1478.

Pavlenko, Ya., Zapatero Osorio, M. R., \& Rebolo, R., 2000, A\&A, in press.

Perryman, M. A. C., et al. 1997, A\&A, 323, L49.

Rebolo, R., Zapatero Osorio, M. R., Madruga, S., Béjar, V. J. S., Arribas, S., \& Licandro, J. 1998, Science, 282, 1309. 
Reid, I. N., et al. 1999, ApJ, 521, 613.

Reid, I. N., Kirkpatrick, J. D., Gizis, J. E., Dahn, C. C., Monet, D. G., Williams, R. J., Liebert, J., \& Burgasser, A. J. 2000, AJ, 119, 369.

Ruiz, M. T., Leggett, S. K., \& Allard, F., 1997, ApJ, 491, L107.

Strauss, M. A., et al. 1999, ApJL, 522, L61.

Tinney, C. G. 1996, MNRAS, 281, 644.

Tinney, C. G., Mould, J. R.., \& Reid, I. N., 1993, AJ, 105, 1045.

Tokunaga, A. T., \& Kobayashi, N. 1999, AJ, 117, 1010.

Tsuji, T., Ohnaka, K., \& Aoki, W., 1996, A\&A, 305, L1.

Tsvetanov, Z., et al. 2000, ApJ, 531, L61.

van Altena, W. F., Lee, J. T., \& Hoffleit, E. D. 1995, The General Catalogue of Trigonometric Stellar Parallaxes (4th ed.; Schenectady: Davis). 
Fig. 1. - Finder charts for each of the $67 \mathrm{~L}$ dwarfs listed in Table 1. For each object, two views are shown - the Digitized Sky Survey (DSS) image on the left and the 2MASS $K_{s}$ image on the right. Each view is to the same scale, five arcminutes on a side with north up and east to the left. The $\mathrm{L}$ dwarf is marked with a box on the 2MASS image, and a box at the same position is also shown on the DSS image. Note that the DSS image is centered on the position of the 2MASS object but that the 2MASS image does not always cover the full $5 \times 5$ arcminute field.

Fig. 2.- Spectra of all 67 new L dwarfs. The flux scale is in units of $F_{\lambda}$ normalized to one at 8250 A. Integral offsets have been added to the flux scale to separate the spectra vertically. Names for the 2MASS objects have been abbreviated.

Fig. 3.- Spectral ratios vs. spectral subclass for the new L dwarfs from this paper (Table 2) together with the $25 \mathrm{~L}$ dwarfs from Paper I: a) CrH-a, b) Rb-b/TiO-b, c) Cs-a/VO-b, and d) Color-d. The primary standards from Paper I are shown as large dots; new objects from this paper are shown with small dots. The spectra with lower signal-to-noise are shown with crosses in panels a) and d).

Fig. 4.- a-b) Detailed spectra of 9 new L dwarfs showing $\mathrm{H} \alpha$ emission. c) Detailed spectra of another 4 new L dwarfs showing possible $\mathrm{H} \alpha$ emission at our limit of detectability.

Fig. 5.- a) $\mathrm{H} \alpha$ equivalent widths as a function of spectral subclass for the $67 \mathrm{~L}$ dwarfs from this paper and the $25 \mathrm{~L}$ dwarfs from Paper I. Open circles denote objects having a detected $\mathrm{H} \alpha$ emission line. Downward arrows denote the upper limits to the $\mathrm{H} \alpha \mathrm{EW}$ for those objects where no line was detected. To display the data more clearly, some of the points have been given slight offsets along the x-axis. b) Percentage of $\mathrm{L}$ dwarfs showing $\mathrm{H} \alpha$ emission as a function of spectral subclass. The only objects used in this computation are those where an $\mathrm{H} \alpha$ equivalent width of $2 \AA$ or more would be detectable. Points have been binned into integer subtypes where L0 and L0.5 dwarfs have been combined into the L0 bin, L1 and L1.5 dwarfs combined into the L1 bin, etc.

Fig. 6. - a) Detailed spectra of 16 new L dwarfs showing lithium absorption. b) Detailed spectra of another 4 new L dwarfs showing possible lithium absorption at our limit of detectability.

Fig. 7.- a) Li I equivalent widths as a function of spectral subclass for the $67 \mathrm{~L}$ dwarfs from this paper and the $25 \mathrm{~L}$ dwarfs from Paper I. Open circles denote objects having a detected Li I absorption line. Downward arrows denote the upper limits to the Li I EW for those objects where no line was detected. To display the data more clearly, some of the points have been given slight offsets along the x-axis. b) Percentage of L dwarfs showing Li I absorption as a function of spectral subclass. The only objects used in this computation are those where a Li I equivalent width of 4 $\AA$ or more would be detectable. Points have been binned into integer subtypes where L0 and L0.5 dwarfs have been combined into the L0 bin, L1 and L1.5 dwarfs combined into the L1 bin, etc. c) Median Li strength as a function of spectral class for those objects where lithium absorption was detected. For spectral subclasses having no objects with detected lithium, no point is plotted. Note the drop in lithium line strength at the latest L types. 
Fig. 8.- Absolute magnitude vs. spectral subclass: a) $M_{J}$ vs. subclass, b) $M_{K_{s}}$ vs. subclass. The second-order fits to each set of data points (given by equations 1 and 2) are also plotted. Absolute magnitudes of two T dwarfs, Gl 229B and Gl 570D, are indicated by the arrows at the bottom right of each panel. Note that in panel a), the faintest L dwarf (Gl 584C) is only 0.4 mag brighter at $J$ than the $\mathrm{T}$ dwarf Gl 229B. In panel b), however, this faintest $\mathrm{L}$ dwarf is 2.6 mag brighter at $K_{s}$ than $\mathrm{Gl} 229 \mathrm{~B}$, demonstrating the profound influence that methane has on reshaping the near-infrared spectral energy distribution in the $\mathrm{T}$ dwarfs.

Fig. 9.- $J-H$ vs. $H-K_{s}$ for $\mathrm{M}, \mathrm{L}$, and $\mathrm{T}$ dwarfs. M dwarfs (solid circles) are taken from Leggett (1992) and Gizis et al. (2000). L dwarfs (open circles) are taken from this paper, Paper I, and Gizis et al. T dwarfs (open stars) are taken from Matthews et al. (1996), Strauss et al. (1999), Burgasser et al. (1999, 2000a, 2000c), and Tsvetanov et al. (2000). L dwarfs having photometric errors of 0.10 mag or larger in either $J, H$, or $K_{s}$ are shown as small open circles, while those with better photometry are shown as large open circles. Tracks for dwarfs (solid line) and giants (dashed line) from Bessell \& Brett (1988) are also plotted. Note that the latest L dwarfs lie in the upper right hand quadrant of this figure, diagonally opposite their slightly cooler counterparts, the $\mathrm{T}$ dwarfs at lower left.

Fig. 10.- Near-infrared color vs. spectral class for late-M through late-L dwarfs: a) $J-K_{s}$, b) $J-H$, c) $H-K_{s}$. Solid circles are plotted for the averages listed in Table 5. Open circles are weighted averages which give a higher weight to colors with smaller measurement errors. See text for details. All data shown here are from 2MASS and are taken from this paper, Paper I, and Gizis et al. (2000).

Fig. 11.- Comparison of absolute magnitude versus spectral type to absolute magnitude versus $J-K_{s}$ color for dwarfs of type M9 through L8. a-b) $M_{J}$, c-d) $M_{K s}$, where panels a) and c) are subsections of Figure 8. Note the markedly increased scatter in the color relation compared to the spectral type relation. See text for details. 


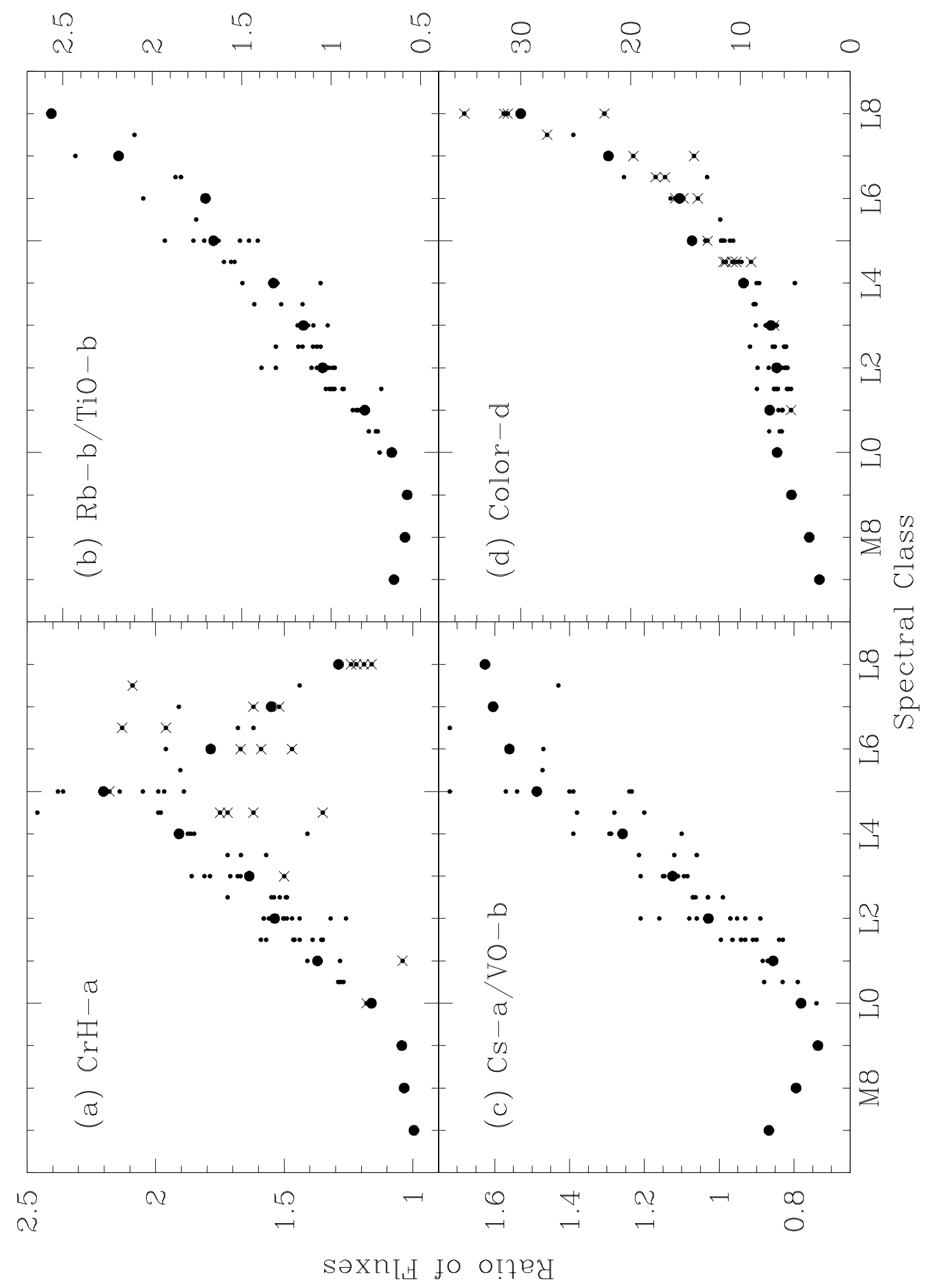

Fig. 3.- 

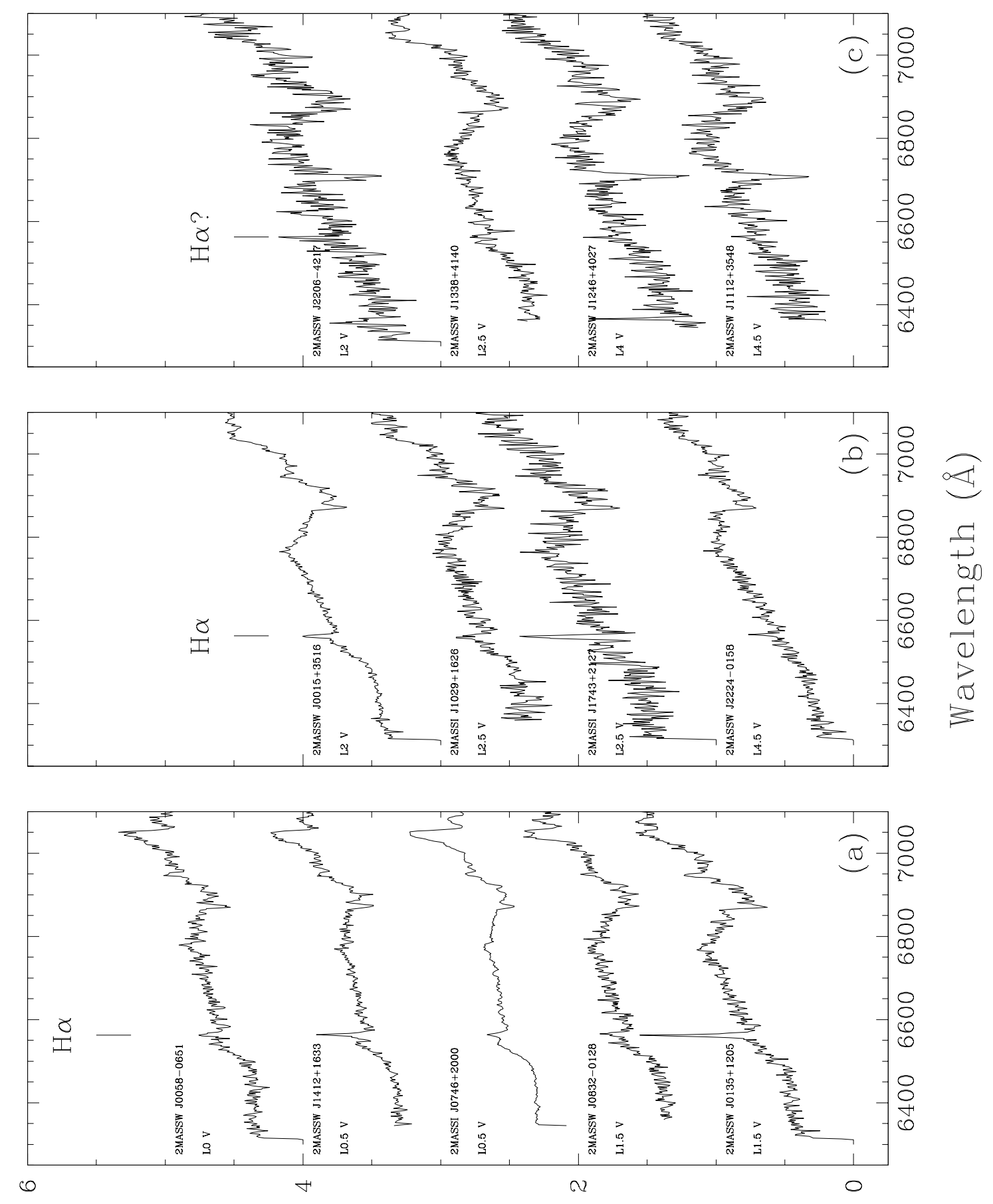

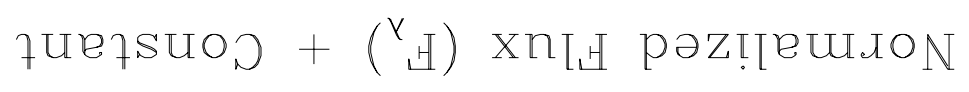

Fig. 4.- 


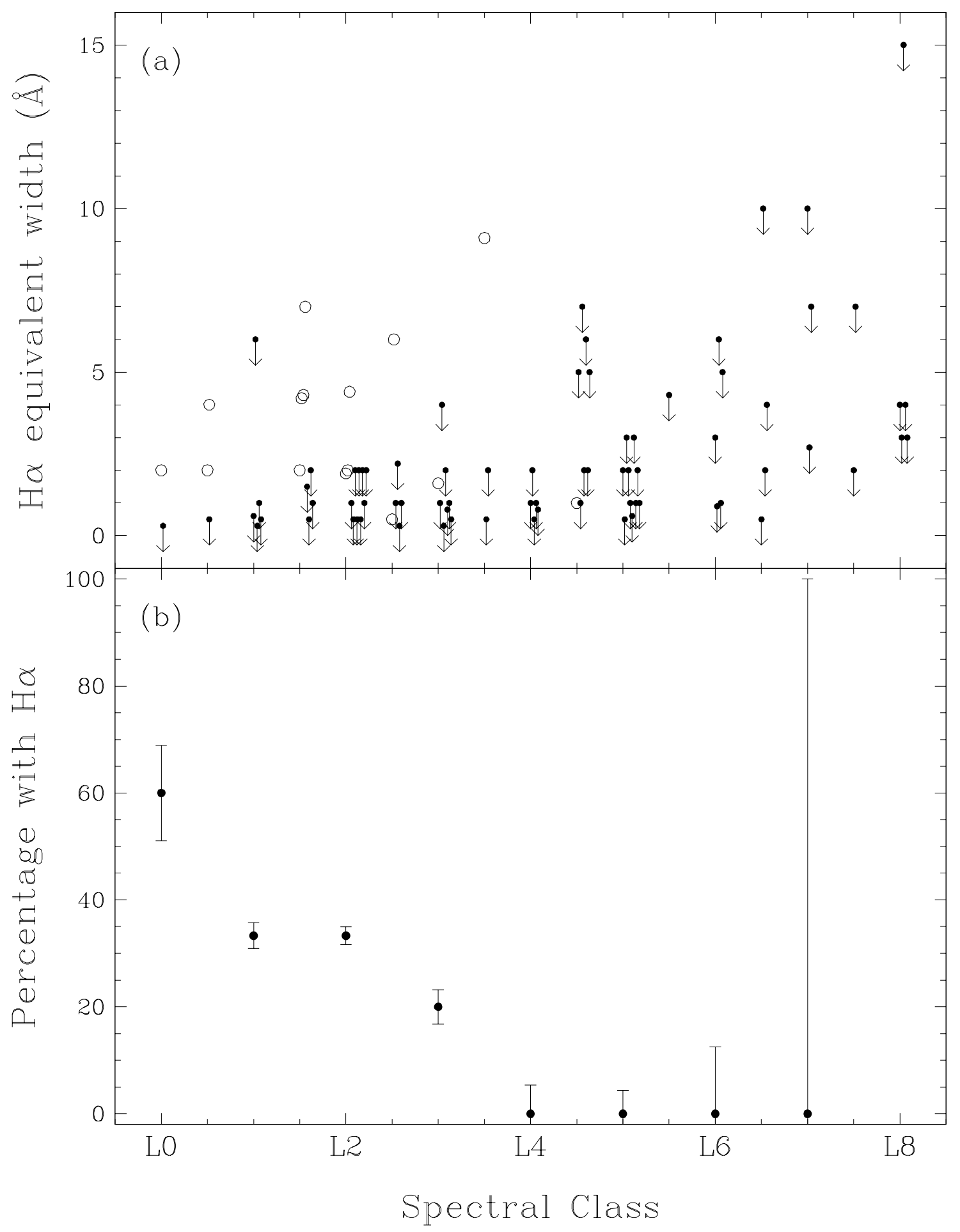

Fig. 5.- 


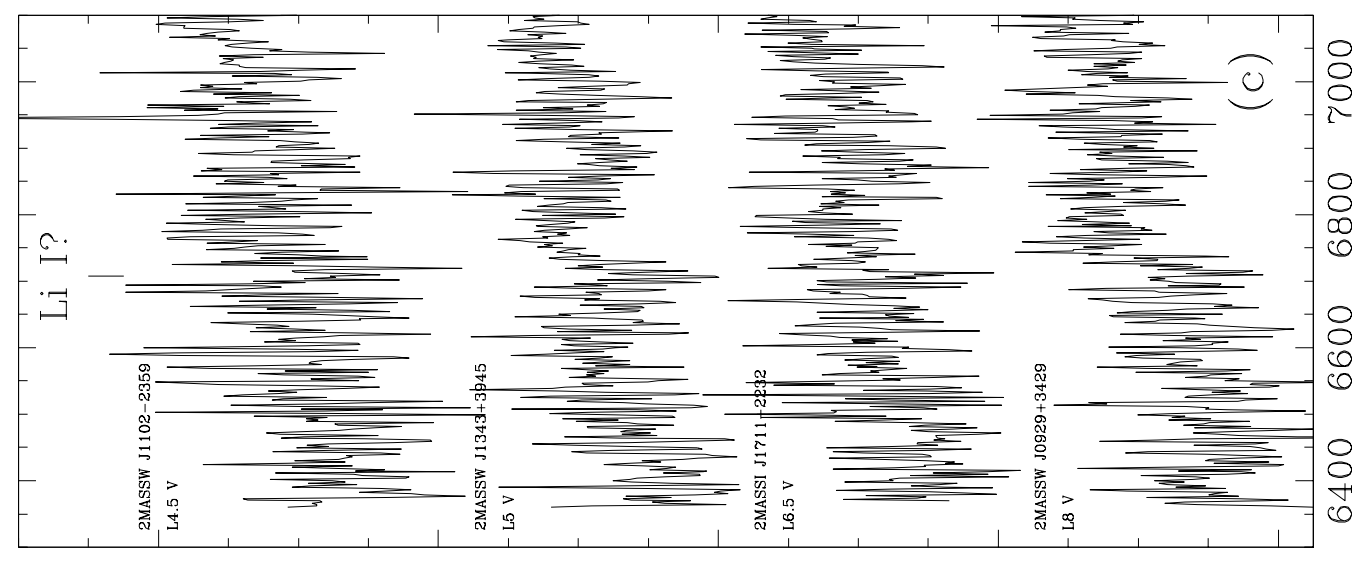
$\infty$
0 ४
N
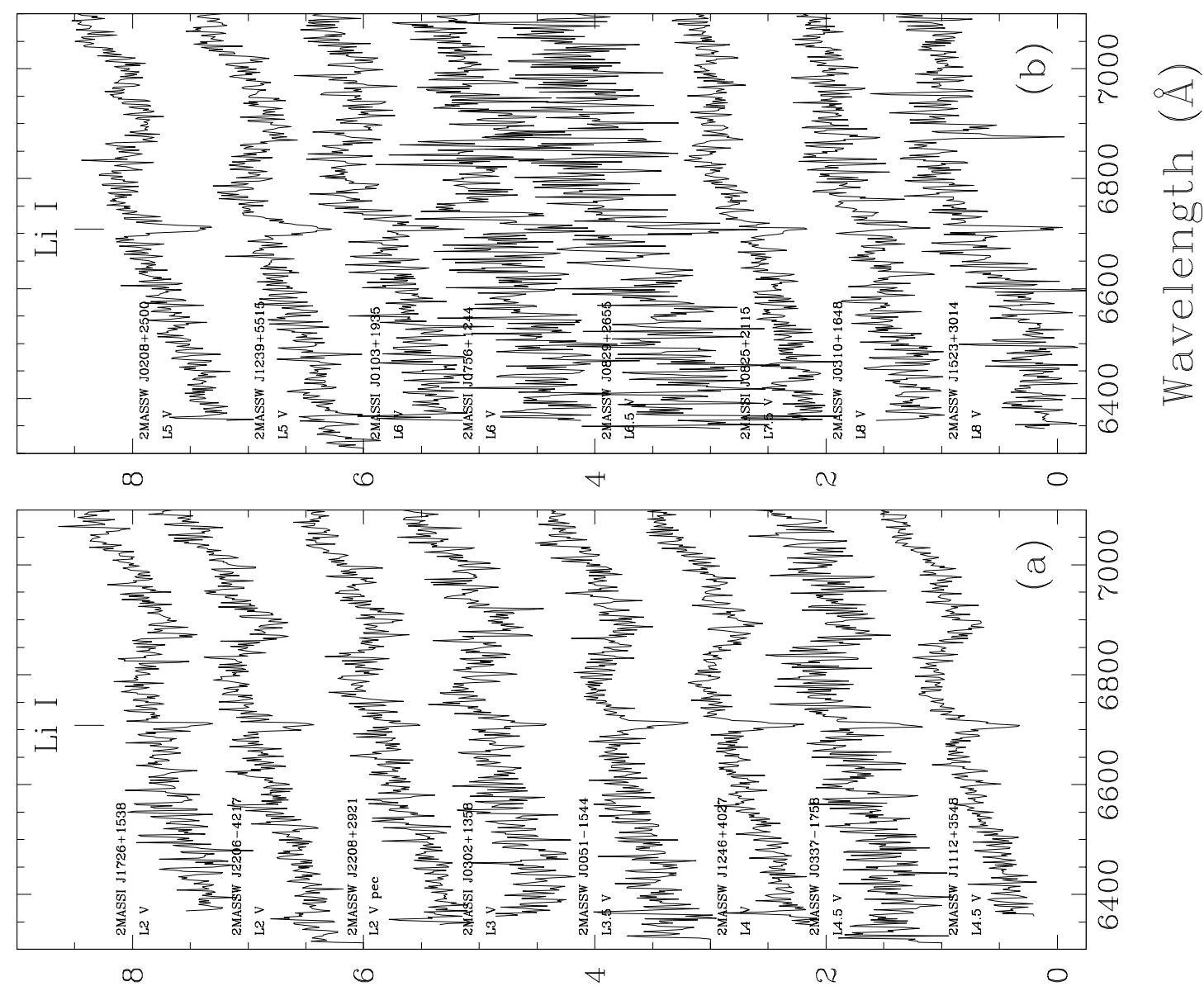

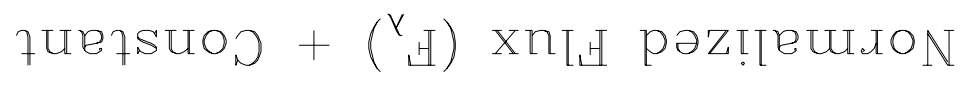

Fig. 6.- 


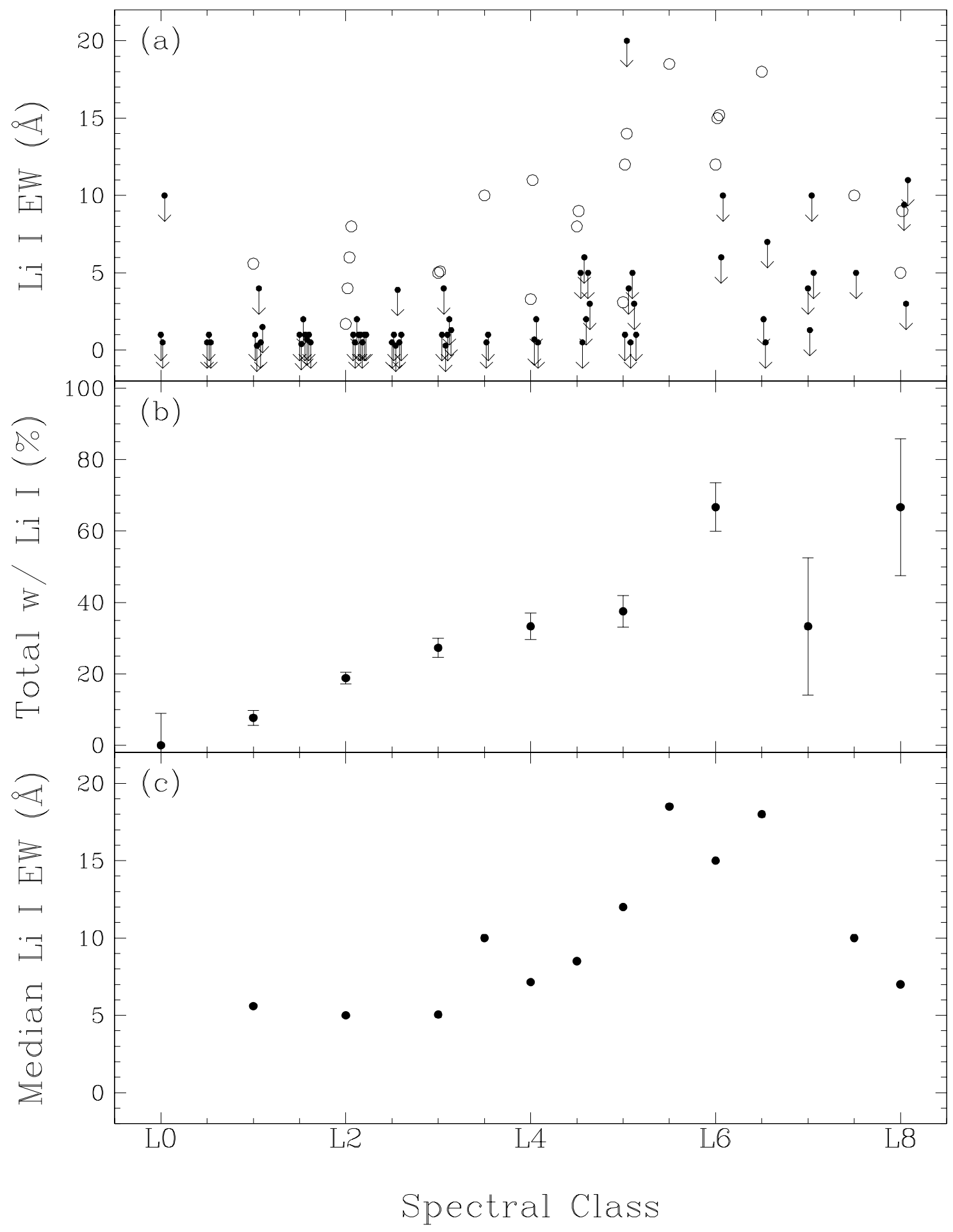

Fig. 7.- 


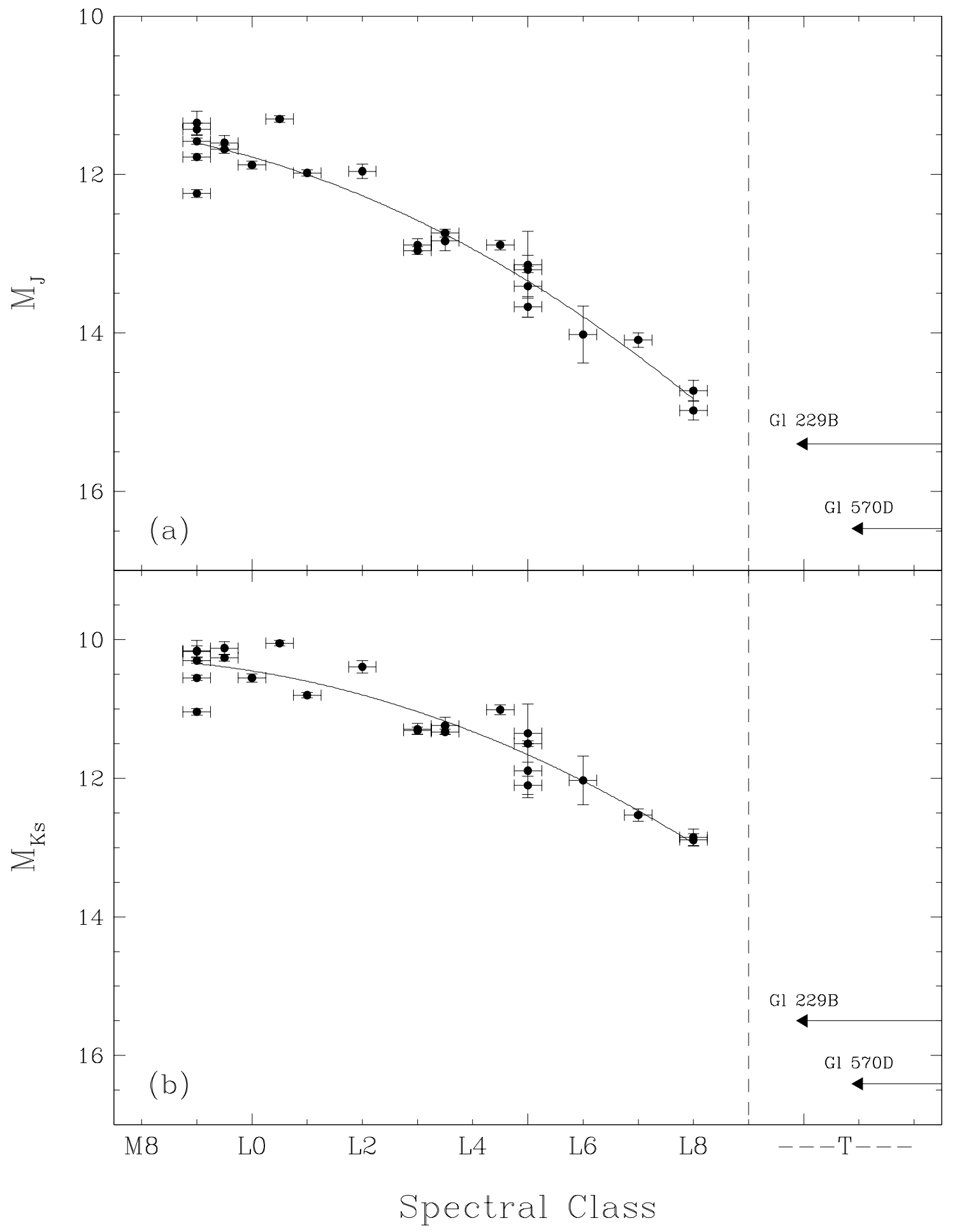

Fig. 8.- 


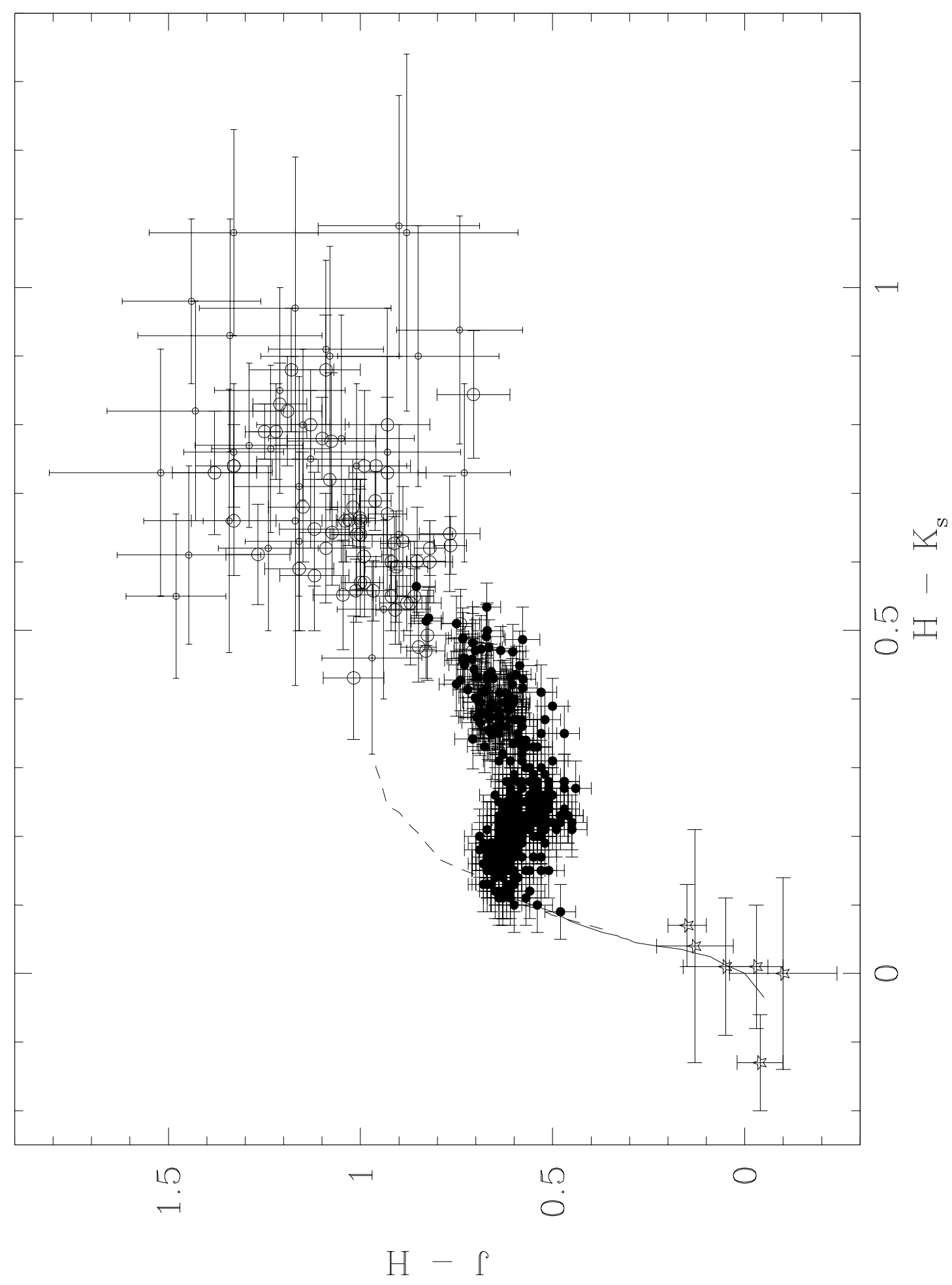

Fig. 9.- 


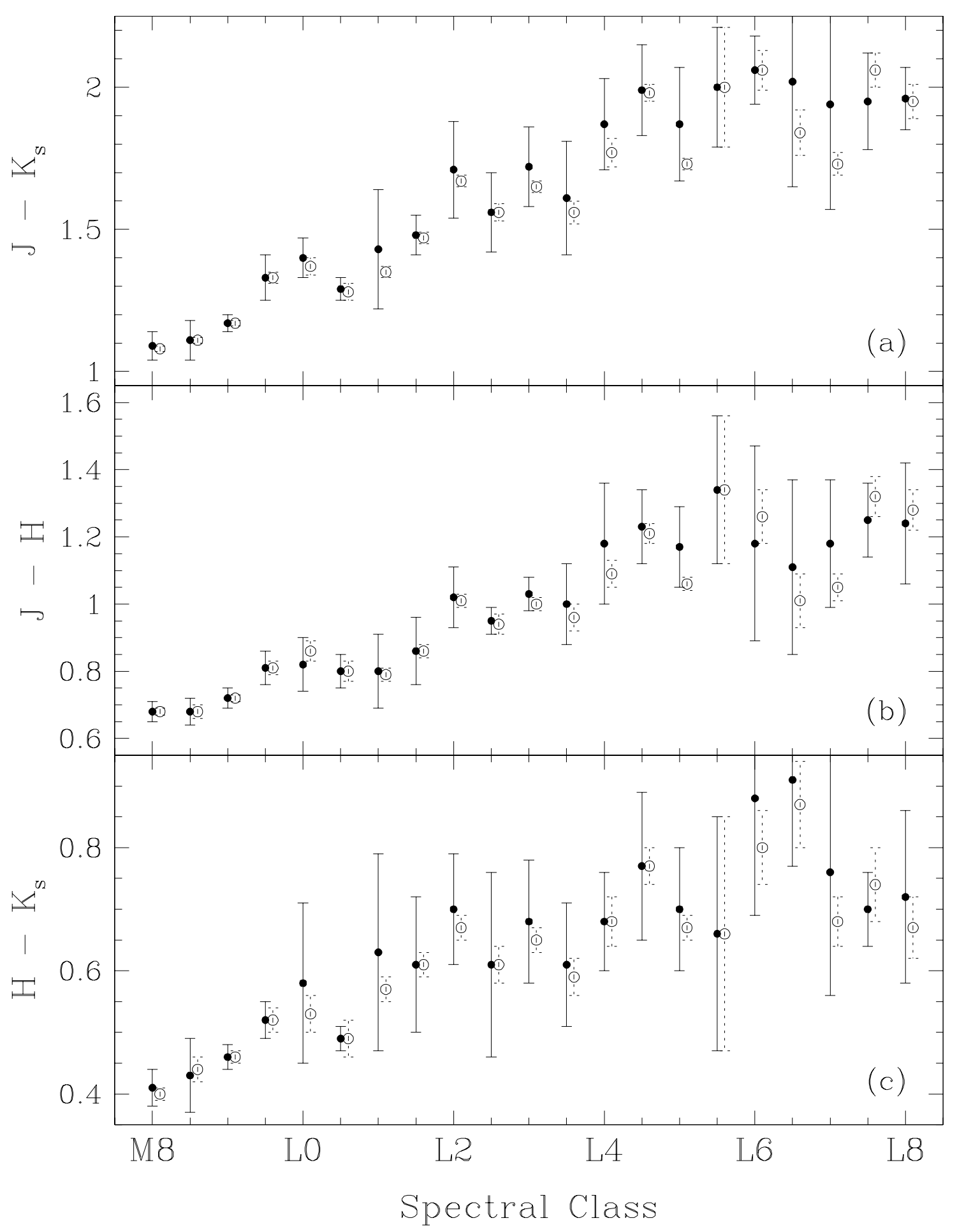

Fig. 10.- 


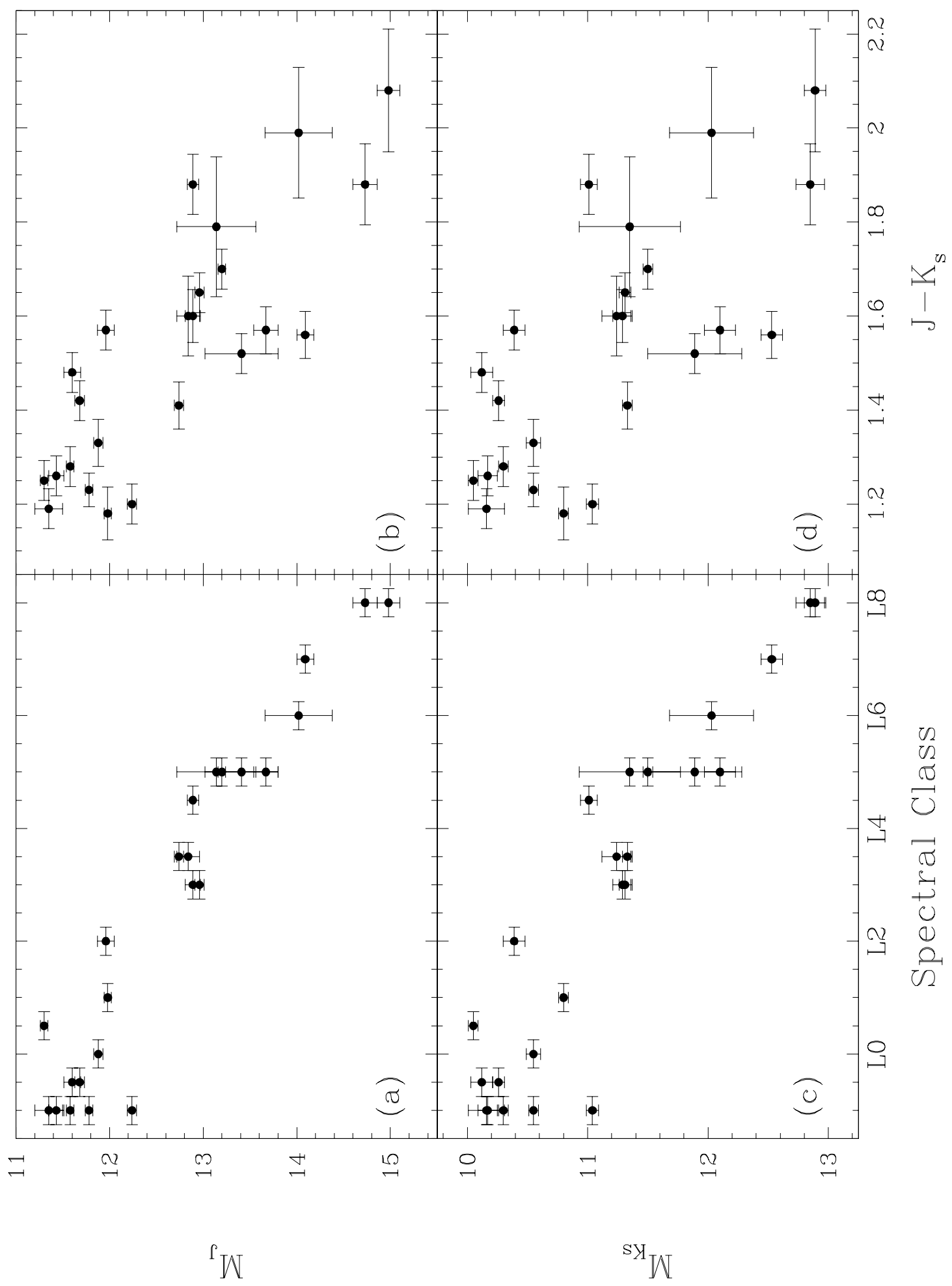

Fig. 11.- 

Table 1. Data for New L Dwarfs

\begin{tabular}{|c|c|c|c|c|c|c|c|c|c|}
\hline $\begin{array}{l}\text { Name of L Dwarf } \\
\text { (1) }\end{array}$ & $\begin{array}{l}J \\
(2)\end{array}$ & $\begin{array}{l}H \\
(3)\end{array}$ & $\begin{array}{l}K_{s} \\
(4)\end{array}$ & $\begin{array}{c}J-K_{s} \\
(5)\end{array}$ & $\begin{array}{c}J-H \\
(6)\end{array}$ & $\begin{array}{c}H-K_{s} \\
(7)\end{array}$ & $\begin{array}{c}\text { Telescope } \\
\text { (8) }\end{array}$ & $\begin{array}{l}\text { Obs. Date } \\
\text { (UT) } \\
\text { (9) }\end{array}$ & $\begin{array}{c}\text { Exposure } \\
\text { Time (sec) } \\
(10)\end{array}$ \\
\hline 2MASSW J0015447+351603 & $13.82 \pm 0.04$ & $12.81 \pm 0.03$ & $12.24 \pm 0.03$ & $1.58 \pm 0.05$ & $1.00 \pm 0.05$ & $0.57 \pm 0.05$ & Keck & 1999 Jul 17 & 1200 \\
\hline 2MASSI J0028394+150141 & $16.49 \pm 0.14$ & $15.33 \pm 0.10$ & $14.62 \pm 0.13$ & $1.87 \pm 0.19$ & $1.16 \pm 0.17$ & $0.71 \pm 0.16$ & Keck & $\begin{array}{l}1998 \text { Dec } 14 \\
1998 \text { Dec } 16\end{array}$ & $\begin{array}{l}3600 \\
2400\end{array}$ \\
\hline 2MASSW J0030300-145033 & $16.79 \pm 0.16$ & $15.36 \pm 0.09$ & $14.38 \pm 0.08$ & $2.41 \pm 0.18$ & $1.44 \pm 0.18$ & $0.98 \pm 0.12$ & Keck & 1999 Jul 18 & 2400 \\
\hline 2MASSW J0036159+182110 & $12.44 \pm 0.04$ & $11.58 \pm 0.03$ & $11.03 \pm 0.03$ & $1.41 \pm 0.05$ & $0.86 \pm 0.05$ & $0.55 \pm 0.04$ & Keck & $\begin{array}{l}1998 \text { Dec } 14 \\
1998 \text { Dec } 16\end{array}$ & $\begin{array}{r}1800 \\
900\end{array}$ \\
\hline 2MASSW J0051107-154417 & $15.23 \pm 0.05$ & $14.15 \pm 0.04$ & $13.42 \pm 0.05$ & $1.81 \pm 0.07$ & $1.08 \pm 0.07$ & $0.72 \pm 0.06$ & Keck & 1999 Jul 18 & 1200 \\
\hline 2MASSW J0058425-065123 & $14.32 \pm 0.03$ & $13.45 \pm 0.03$ & $12.91 \pm 0.04$ & $1.41 \pm 0.05$ & $0.88 \pm 0.04$ & $0.54 \pm 0.04$ & Keck & 1999 Jul 17 & 900 \\
\hline 2MASSI J0103320+193536 & $16.26 \pm 0.09$ & $14.88 \pm 0.06$ & $14.15 \pm 0.07$ & $2.11 \pm 0.11$ & $1.38 \pm 0.11$ & $0.73 \pm 0.09$ & Keck & $\begin{array}{l}1998 \text { Dec } 14 \\
1998 \text { Dec } 16\end{array}$ & $\begin{array}{l}2400 \\
2400\end{array}$ \\
\hline 2MASSW J0135358+120522 & $14.43 \pm 0.04$ & $13.44 \pm 0.03$ & $12.86 \pm 0.03$ & $1.57 \pm 0.05$ & $0.99 \pm 0.05$ & $0.57 \pm 0.05$ & Keck & 1999 Jul 17 & 900 \\
\hline 2MASSW J0205034+125142 & $15.69 \pm 0.06$ & $14.47 \pm 0.05$ & $13.68 \pm 0.05$ & $2.01 \pm 0.08$ & $1.22 \pm 0.08$ & $0.79 \pm 0.07$ & Keck & 1998 Dec 14 & 2400 \\
\hline 2MASSW J0208183+254253 & $14.02 \pm 0.03$ & $13.11 \pm 0.04$ & $12.58 \pm 0.04$ & $1.44 \pm 0.05$ & $0.91 \pm 0.05$ & $0.53 \pm 0.05$ & Keck & 1999 Jul 17 & 600 \\
\hline 2MASSW J0208236+273740 & $15.70 \pm 0.07$ & $14.55 \pm 0.06$ & $13.87 \pm 0.06$ & $1.83 \pm 0.09$ & $1.15 \pm 0.09$ & $0.68 \pm 0.08$ & Keck & 1998 Dec 15 & 2400 \\
\hline 2MASSW J0208549+250048 & $16.24 \pm 0.10$ & $15.00 \pm 0.09$ & $14.38 \pm 0.08$ & $1.86 \pm 0.13$ & $1.24 \pm 0.13$ & $0.62 \pm 0.12$ & Keck & 1998 Dec 15 & 2400 \\
\hline 2MASSI J0224367+253704 & $16.55 \pm 0.11$ & $15.42 \pm 0.09$ & $14.67 \pm 0.09$ & $1.88 \pm 0.14$ & $1.13 \pm 0.14$ & $0.75 \pm 0.13$ & Keck & $\begin{array}{l}1998 \text { Dec } 15 \\
1998 \text { Dec } 16\end{array}$ & $\begin{array}{l}2400 \\
2400\end{array}$ \\
\hline 2MASSI J0302012+135814 & $16.55 \pm 0.12$ & $15.46 \pm 0.09$ & $14.55 \pm 0.09$ & $2.00 \pm 0.15$ & $1.09 \pm 0.15$ & $0.91 \pm 0.13$ & Keck & $\begin{array}{l}1998 \text { Dec } 14 \\
1998 \text { Dec } 16\end{array}$ & $\begin{array}{l}2400 \\
1200\end{array}$ \\
\hline 2MASSW J0306268+154514 & $17.12 \pm 0.19$ & $16.24 \pm 0.22$ & $15.16 \pm 0.14$ & $1.96 \pm 0.24$ & $0.88 \pm 0.29$ & $1.08 \pm 0.26$ & Keck & 1998 Dec 24 & 3600 \\
\hline 2MASSW J0309088-194938 & $15.82 \pm 0.06$ & $14.67 \pm 0.06$ & $14.08 \pm 0.07$ & $1.74 \pm 0.09$ & $1.16 \pm 0.09$ & $0.59 \pm 0.09$ & Keck & 1999 Jul 18 & 1200 \\
\hline 2MASSW J0310599+164816 & $16.43 \pm 0.11$ & $14.95 \pm 0.07$ & $14.40 \pm 0.10$ & $2.03 \pm 0.15$ & $1.48 \pm 0.13$ & $0.55 \pm 0.12$ & Keck & $\begin{array}{l}1998 \text { Dec } 15 \\
1998 \text { Dec } 16\end{array}$ & $\begin{array}{l}4800 \\
3600\end{array}$ \\
\hline 2MASSI J0328426+230205 & $16.67 \pm 0.14$ & $15.62 \pm 0.13$ & $14.84 \pm 0.13$ & $1.83 \pm 0.19$ & $1.05 \pm 0.19$ & $0.78 \pm 0.18$ & Keck & $\begin{array}{l}1998 \text { Dec } 15 \\
1998 \text { Dec } 16 \\
1999 \text { Mar } 04\end{array}$ & $\begin{array}{l}3600 \\
3600 \\
2400\end{array}$ \\
\hline 2MASSW J0337036-175807 & $15.60 \pm 0.06$ & $14.39 \pm 0.05$ & $13.57 \pm 0.04$ & $2.03 \pm 0.07$ & $1.21 \pm 0.07$ & $0.83 \pm 0.06$ & Keck & 1999 Jul 17 & 2400 \\
\hline 2MASSI J0409095+210439 & $15.55 \pm 0.07$ & $14.46 \pm 0.06$ & $13.84 \pm 0.06$ & $1.71 \pm 0.09$ & $1.09 \pm 0.09$ & $0.62 \pm 0.08$ & Keck & 1998 Dec 16 & 1200 \\
\hline 2MASSW J0708213+295035 & $16.75 \pm 0.12$ & $15.54 \pm 0.12$ & $14.69 \pm 0.09$ & $2.06 \pm 0.15$ & $1.21 \pm 0.17$ & $0.85 \pm 0.15$ & Keck & 1998 Dec 24 & 2400 \\
\hline 2MASSW J0740096+321203 & $16.17 \pm 0.09$ & $14.84 \pm 0.06$ & $14.18 \pm 0.06$ & $1.99 \pm 0.11$ & $1.33 \pm 0.11$ & $0.66 \pm 0.08$ & Keck & 1998 Dec 14 & 2400 \\
\hline 2MASSI J0746425+200032 & $11.74 \pm 0.03$ & $11.00 \pm 0.04$ & $10.49 \pm 0.03$ & $1.25 \pm 0.04$ & $0.74 \pm 0.05$ & $0.51 \pm 0.05$ & Keck & 1998 Dec 24 & 600 \\
\hline 2MASSI J0753321+291711 & $15.49 \pm 0.05$ & $14.49 \pm 0.05$ & $13.85 \pm 0.06$ & $1.64 \pm 0.08$ & $1.00 \pm 0.07$ & $0.64 \pm 0.08$ & Keck & 1998 Dec 24 & 2400 \\
\hline 2MASSI J0756252+124456 & $16.66 \pm 0.14$ & $15.76 \pm 0.15$ & $14.67 \pm 0.12$ & $1.99 \pm 0.18$ & $0.90 \pm 0.21$ & $1.09 \pm 0.19$ & Keck & $\begin{array}{l}1998 \text { Dec } 15 \\
1998 \text { Dec } 16\end{array}$ & $\begin{array}{l}2400 \\
1200\end{array}$ \\
\hline 2MASSW J0801405+462850 & $16.29 \pm 0.14$ & $15.44 \pm 0.15$ & $14.54 \pm 0.11$ & $1.75 \pm 0.18$ & $0.85 \pm 0.21$ & $0.90 \pm 0.19$ & Keck & 1998 Dec 15 & 2400 \\
\hline 2MASSW J0820299+450031 & $16.29 \pm 0.11$ & $15.00 \pm 0.09$ & $14.23 \pm 0.08$ & $2.06 \pm 0.14$ & $1.29 \pm 0.14$ & $0.77 \pm 0.12$ & Keck & $\begin{array}{l}1998 \text { Dec } 14 \\
1998 \text { Dec } 16\end{array}$ & $\begin{array}{l}3600 \\
2400\end{array}$ \\
\hline 2MASSI J0825196+211552 & $15.12 \pm 0.04$ & $13.79 \pm 0.04$ & $13.05 \pm 0.04$ & $2.07 \pm 0.06$ & $1.33 \pm 0.06$ & $0.74 \pm 0.06$ & Keck & 1998 Dec 14 & 2400 \\
\hline
\end{tabular}


Table 1. (continued)

\begin{tabular}{|c|c|c|c|c|c|c|c|c|c|}
\hline $\begin{array}{c}\text { Name of L Dwarf } \\
\text { (1) }\end{array}$ & $\begin{array}{l}J \\
(2)\end{array}$ & $\begin{array}{l}H \\
(3)\end{array}$ & $\begin{array}{l}K_{s} \\
(4)\end{array}$ & $\begin{array}{c}J-K_{s} \\
(5)\end{array}$ & $\begin{array}{c}J-H \\
(6)\end{array}$ & $\begin{array}{c}H-K_{s} \\
(7)\end{array}$ & $\begin{array}{c}\text { Telescope } \\
\text { (8) }\end{array}$ & $\begin{array}{l}\text { Obs. Date } \\
\text { (UT) } \\
\text { (9) }\end{array}$ & $\begin{array}{c}\text { Exposure } \\
\text { Time (sec) } \\
(10)\end{array}$ \\
\hline 2MASSW J0829066+145622 & $14.72 \pm 0.03$ & $13.79 \pm 0.04$ & $13.12 \pm 0.05$ & $1.60 \pm 0.06$ & $0.93 \pm 0.05$ & $0.67 \pm 0.06$ & Keck & 1998 Dec 15 & 1200 \\
\hline 2MASSW J0829570+265510 & $17.08 \pm 0.20$ & $15.74 \pm 0.14$ & $14.81 \pm 0.10$ & $2.27 \pm 0.30$ & $1.34 \pm 0.24$ & $0.93 \pm 0.17$ & Keck & 1998 Dec 24 & 2400 \\
\hline 2MASSW J0832045-012835 & $14.13 \pm 0.03$ & $13.31 \pm 0.03$ & $12.69 \pm 0.03$ & $1.44 \pm 0.04$ & $0.82 \pm 0.04$ & $0.62 \pm 0.04$ & Keck & 1999 Mar 04 & 1200 \\
\hline 2MASSW J0920122+351742 & $15.59 \pm 0.07$ & $14.66 \pm 0.07$ & $13.93 \pm 0.08$ & $1.66 \pm 0.11$ & $0.93 \pm 0.10$ & $0.73 \pm 0.11$ & Keck & 1998 Dec 24 & 2400 \\
\hline 2MASSW J0928397-160312 & $15.34 \pm 0.05$ & $14.30 \pm 0.04$ & $13.64 \pm 0.05$ & $1.70 \pm 0.07$ & $1.04 \pm 0.06$ & $0.66 \pm 0.06$ & Keck & 1998 Dec 25 & 2400 \\
\hline 2MASSW J0929336+342952 & $16.60 \pm 0.13$ & $15.52 \pm 0.12$ & $14.62 \pm 0.11$ & $1.98 \pm 0.17$ & $1.08 \pm 0.18$ & $0.90 \pm 0.16$ & Keck & $\begin{array}{l}1998 \text { Dec } 15 \\
1998 \text { Dec } 16\end{array}$ & $\begin{array}{l}2400 \\
3600\end{array}$ \\
\hline 2MASSW J0944027+313132 & $15.50 \pm 0.06$ & $14.61 \pm 0.06$ & $13.98 \pm 0.05$ & $1.52 \pm 0.08$ & $0.89 \pm 0.08$ & $0.63 \pm 0.08$ & Keck & 1998 Dec 25 & 2400 \\
\hline 2MASSW J0951054+355801 & $17.29 \pm 0.25$ & $15.77 \pm 0.14$ & $15.04 \pm 0.12$ & $2.25 \pm 0.28$ & $1.52 \pm 0.29$ & $0.73 \pm 0.18$ & Keck & $\begin{array}{l}1998 \text { Dec } 15 \\
1998 \text { Dec } 16\end{array}$ & $\begin{array}{l}3600 \\
3600\end{array}$ \\
\hline 2MASSI J1029216+162652 & $14.31 \pm 0.04$ & $13.35 \pm 0.04$ & $12.61 \pm 0.04$ & $1.70 \pm 0.06$ & $0.96 \pm 0.06$ & $0.74 \pm 0.06$ & Keck & 1998 Dec 14 & 1200 \\
\hline 2MASSW J1035245+250745 & $14.70 \pm 0.04$ & $13.88 \pm 0.04$ & $13.28 \pm 0.04$ & $1.42 \pm 0.06$ & $0.82 \pm 0.06$ & $0.60 \pm 0.06$ & Keck & 1998 Dec 25 & 1200 \\
\hline 2MASSW J1102337-235945 & $17.04 \pm 0.19$ & $15.61 \pm 0.13$ & $14.79 \pm 0.09$ & $2.25 \pm 0.21$ & $1.43 \pm 0.23$ & $0.82 \pm 0.16$ & Keck & $\begin{array}{l}1998 \text { Dec } 14 \\
1998 \text { Dec } 16\end{array}$ & $\begin{array}{l}2400 \\
2400\end{array}$ \\
\hline 2MASSW J1112257+354813 & $14.57 \pm 0.04$ & $13.47 \pm 0.04$ & $12.69 \pm 0.05$ & $1.88 \pm 0.06$ & $1.10 \pm 0.06$ & $0.78 \pm 0.06$ & Keck & $\begin{array}{l}1998 \text { Dec } 14 \\
1999 \text { Mar } 04 \\
1999 \text { Mar } 05\end{array}$ & $\begin{array}{l}1200 \\
1200 \\
3600\end{array}$ \\
\hline 2MASSW J1123556+412228 & $16.07 \pm 0.08$ & $15.14 \pm 0.08$ & $14.34 \pm 0.06$ & $1.73 \pm 0.10$ & $0.93 \pm 0.11$ & $0.80 \pm 0.10$ & Keck & 1998 Dec 25 & 2400 \\
\hline 2MASSW J1239272+551537 & $14.67 \pm 0.03$ & $13.54 \pm 0.04$ & $12.74 \pm 0.03$ & $1.93 \pm 0.05$ & $1.13 \pm 0.05$ & $0.80 \pm 0.05$ & Keck & 1999 Jul 17 & 1200 \\
\hline 2MASSW J1246467+402715 & $15.00 \pm 0.04$ & $13.98 \pm 0.04$ & $13.30 \pm 0.04$ & $1.70 \pm 0.06$ & $1.02 \pm 0.06$ & $0.68 \pm 0.06$ & Keck & 1998 Dec 24 & 600 \\
\hline 2MASSI J1332286+263508 & $16.11 \pm 0.10$ & $15.10 \pm 0.09$ & $14.36 \pm 0.08$ & $1.75 \pm 0.13$ & $1.01 \pm 0.13$ & $0.74 \pm 0.12$ & Keck & 1998 Dec 25 & 1200 \\
\hline 2MASSW J1338261+414034 & $14.22 \pm 0.03$ & $13.30 \pm 0.03$ & $12.75 \pm 0.04$ & $1.47 \pm 0.05$ & $0.92 \pm 0.04$ & $0.55 \pm 0.05$ & Keck & 1998 Dec 14 & 1200 \\
\hline 2MASSW J1343167+394508 & $16.18 \pm 0.08$ & $14.85 \pm 0.06$ & $14.11 \pm 0.06$ & $2.07 \pm 0.10$ & $1.33 \pm 0.10$ & $0.74 \pm 0.08$ & Keck & 1998 Dec 14 & 1200 \\
\hline 2MASSW J1411175+393636 & $14.68 \pm 0.04$ & $13.81 \pm 0.07$ & $13.27 \pm 0.05$ & $1.41 \pm 0.06$ & $0.87 \pm 0.08$ & $0.54 \pm 0.09$ & Keck & 1999 Mar 05 & 1800 \\
\hline 2MASSW J1412244+163312 & $13.89 \pm 0.04$ & $13.06 \pm 0.03$ & $12.59 \pm 0.03$ & $1.30 \pm 0.05$ & $0.83 \pm 0.05$ & $0.47 \pm 0.04$ & Keck & 1998 Dec 25 & 1200 \\
\hline 2MASSW J1438549-130910 & $15.53 \pm 0.05$ & $14.52 \pm 0.05$ & $13.88 \pm 0.06$ & $1.65 \pm 0.08$ & $1.01 \pm 0.07$ & $0.64 \pm 0.08$ & Keck & 1999 Mar 04 & 2400 \\
\hline 2MASSW J1449378+235537 & $15.80 \pm 0.08$ & $15.07 \pm 0.09$ & $14.34 \pm 0.10$ & $1.46 \pm 0.13$ & $0.73 \pm 0.12$ & $0.73 \pm 0.13$ & Palomar & 1998 Jul 19 & 1200 \\
\hline 2MASSW J1507476-162738 & $12.82 \pm 0.03$ & $11.90 \pm 0.03$ & $11.30 \pm 0.03$ & $1.52 \pm 0.04$ & $0.92 \pm 0.04$ & $0.60 \pm 0.04$ & Keck & $\begin{array}{l}1998 \text { Dec } 24 \\
1999 \text { Mar } 05\end{array}$ & $\begin{array}{r}900 \\
1800\end{array}$ \\
\hline 2MASSW J1523226+301456 & $16.32 \pm 0.11$ & $15.00 \pm 0.07$ & $14.24 \pm 0.07$ & $2.09 \pm 0.13$ & $1.33 \pm 0.13$ & $0.76 \pm 0.10$ & Keck & $\begin{array}{l}1998 \text { Dec } 24 \\
1998 \text { Dec } 25 \\
\text { 1999 Mar } 04 \\
\text { 1999 Mar } 31\end{array}$ & $\begin{array}{l}3600 \\
4600 \\
3600 \\
4800\end{array}$ \\
\hline 2MASSI J1526140+204341 & $15.62 \pm 0.07$ & $14.50 \pm 0.06$ & $13.92 \pm 0.06$ & $1.70 \pm 0.09$ & $1.12 \pm 0.09$ & $0.58 \pm 0.08$ & Palomar & 1998 Sep 20 & 1200 \\
\hline 2MASSI J1600054+170832 & $16.10 \pm 0.10$ & $15.13 \pm 0.08$ & $14.67 \pm 0.12$ & $1.43 \pm 0.16$ & $0.97 \pm 0.13$ & $0.46 \pm 0.14$ & Keck & 1999 Jul 16 & 1200 \\
\hline 2MASSW J1615441+355900 & $14.55 \pm 0.04$ & $13.55 \pm 0.04$ & $12.89 \pm 0.05$ & $1.67 \pm 0.06$ & $1.00 \pm 0.06$ & $0.66 \pm 0.06$ & Keck & 1999 Jul 16 & 1200 \\
\hline 2MASSI J1656188+283506 & $17.10 \pm 0.20$ & $15.93 \pm 0.15$ & $14.96 \pm 0.16$ & $2.14 \pm 0.26$ & $1.17 \pm 0.25$ & $0.97 \pm 0.22$ & Keck & $\begin{array}{c}1998 \text { Aug } 13 \\
1999 \text { Jul } 17\end{array}$ & $\begin{array}{l}2400 \\
1200\end{array}$ \\
\hline
\end{tabular}


TABLE 1. (continued)

\begin{tabular}{|c|c|c|c|c|c|c|c|c|c|}
\hline $\begin{array}{c}\text { Name of L Dwarf } \\
\text { (1) }\end{array}$ & $\begin{array}{l}J \\
(2)\end{array}$ & $\begin{array}{l}H \\
(3)\end{array}$ & $\begin{array}{l}K_{s} \\
(4)\end{array}$ & $\begin{array}{c}J-K_{s} \\
(5)\end{array}$ & $\begin{array}{c}J-H \\
(6)\end{array}$ & $\begin{array}{c}H-K_{s} \\
(7)\end{array}$ & $\begin{array}{c}\text { Telescope } \\
\text { (8) }\end{array}$ & $\begin{array}{l}\text { Obs. Date } \\
\text { (UT) } \\
\text { (9) }\end{array}$ & $\begin{array}{c}\text { Exposure } \\
\text { Time (sec) } \\
\quad(10)\end{array}$ \\
\hline 2MASSI J1711457+223204 & $17.10 \pm 0.19$ & $15.77 \pm 0.11$ & $14.69 \pm 0.10$ & $2.41 \pm 0.21$ & $1.33 \pm 0.22$ & $1.08 \pm 0.15$ & Keck & 1998 Aug 13 & 2400 \\
\hline 2MASSI J1726000+153819 & $15.65 \pm 0.07$ & $14.46 \pm 0.06$ & $13.64 \pm 0.05$ & $2.01 \pm 0.09$ & $1.19 \pm 0.09$ & $0.82 \pm 0.08$ & Keck & 1998 Aug 13 & 2400 \\
\hline 2MASSW J1728114+394859 & $15.96 \pm 0.08$ & $14.78 \pm 0.07$ & $13.90 \pm 0.05$ & $2.07 \pm 0.10$ & $1.18 \pm 0.11$ & $0.88 \pm 0.09$ & Keck & 1999 Jul 16 & 2400 \\
\hline 2MASSW J1743415+212707 & $15.80 \pm 0.09$ & $14.78 \pm 0.07$ & $14.29 \pm 0.10$ & $1.51 \pm 0.13$ & $1.02 \pm 0.11$ & $0.49 \pm 0.12$ & Keck & $1999 \mathrm{Jul} 17$ & 1200 \\
\hline 2MASSW J1841086+311727 & $16.12 \pm 0.10$ & $14.97 \pm 0.07$ & $14.18 \pm 0.08$ & $1.95 \pm 0.13$ & $1.15 \pm 0.12$ & $0.80 \pm 0.11$ & Keck & 1999 Jul 18 & 2400 \\
\hline 2MASSI J2054358+151904 & $16.51 \pm 0.13$ & $15.58 \pm 0.14$ & $14.82 \pm 0.16$ & $1.69 \pm 0.21$ & $0.93 \pm 0.19$ & $0.76 \pm 0.21$ & Keck & 1998 Aug 13 & 2400 \\
\hline 2MASSI J2057153+171515 & $16.11 \pm 0.11$ & $15.21 \pm 0.09$ & $14.57 \pm 0.13$ & $1.54 \pm 0.17$ & $0.90 \pm 0.14$ & $0.64 \pm 0.16$ & Keck & $1999 \mathrm{Jul} 17$ & 1200 \\
\hline 2MASSW J2101154+175658 & $16.87 \pm 0.19$ & $15.70 \pm 0.14$ & $15.04 \pm 0.19$ & $1.83 \pm 0.27$ & $1.17 \pm 0.24$ & $0.66 \pm 0.24$ & Keck & 1998 Aug 13 & 3600 \\
\hline 2MASSW J2206540-421721 & $15.57 \pm 0.07$ & $14.48 \pm 0.06$ & $13.60 \pm 0.06$ & $1.97 \pm 0.09$ & $1.09 \pm 0.09$ & $0.88 \pm 0.08$ & Keck & 1999 Jul 17 & 1200 \\
\hline 2MASSW J2208136+292121 & $15.82 \pm 0.09$ & $14.83 \pm 0.08$ & $14.09 \pm 0.08$ & $1.73 \pm 0.12$ & $0.99 \pm 0.12$ & $0.74 \pm 0.11$ & Keck & 1998 Dec 24 & 2150 \\
\hline & & & & & & & & 1998 Dec 25 & 2400 \\
\hline 2MASSW J2224438-015852 & $14.05 \pm 0.03$ & $12.80 \pm 0.03$ & $12.02 \pm 0.03$ & $2.04 \pm 0.04$ & $1.25 \pm 0.04$ & $0.79 \pm 0.04$ & Keck & 1999 Jul 16 & 1200 \\
\hline
\end{tabular}

${ }^{2}$ Source designations for 2 MASS discoveries are given as "2MAS $x$ Jhhmmss[.]s \pm ddmmss." The " $x$ " in the prefix will vary depending upon the catalog from which the object was taken; "P" is used for objects discovered in the prototype data, "SW" is used for objects taken from the actual survey's working database, and "SI" is used for objects taken from one of the 2MASS incremental releases. The suffix conforms to IAU nomenclature convention and is the sexigesimal R.A. and decl. at J2000 equinox. 

Table 2. Additional Data for New L Dwarfs

\begin{tabular}{|c|c|c|c|c|c|c|c|c|c|c|}
\hline $\begin{array}{l}\text { Name of L Dwarf } \\
\text { (1) }\end{array}$ & $\begin{array}{c}\text { CrH-a } \\
(2)\end{array}$ & $\begin{array}{c}\text { Rb-b/TiO-b } \\
\text { (3) }\end{array}$ & $\begin{array}{c}\mathrm{Cs}-\mathrm{a} / \mathrm{VO}-\mathrm{b} \\
\text { (4) }\end{array}$ & $\begin{array}{l}\text { Color-d } \\
\text { (5) }\end{array}$ & $\begin{array}{l}\text { K I Fit } \\
(6)\end{array}$ & $\begin{array}{l}\text { Oxide } \\
\text { Fit } \\
(7)\end{array}$ & $\begin{array}{l}\text { Spectral } \\
\text { Type } \\
(8)\end{array}$ & $\begin{array}{c}\mathrm{H} \alpha \\
\mathrm{EW}(\AA) \\
(9)\end{array}$ & $\begin{array}{c}\text { Li I } \\
\text { EW }(\AA) \\
(10)\end{array}$ & $\begin{array}{c}\text { Est. Dist. } \\
\text { (pc) } \\
\text { (11) }\end{array}$ \\
\hline 2MASSW J0015447+351603 & $1.47(1-2)$ & $1.07(2)$ & $0.97(2)$ & $5.77(-)$ & $(2)$ & - & L2 $\mathrm{V}$ & 2 & $<0.5$ & 20 \\
\hline 2MASSI J0028394+150141 & $2.84(5)$ & - & - & $10.38(5)$ & (4) & $(5)$ & $\mathrm{L} 4.5 \mathrm{~V}$ & $<2$ & $<5$ & 45 \\
\hline 2MASSW J0030300-145033 & $1.62(7)$ & - & - & $14.23(5)$ & - & (8) & $\mathrm{L} 7 \mathrm{~V}$ & $<10$ & $<5$ & 28 \\
\hline 2MASSW J0036159+182110 & $1.72(3-4)$ & $1.16(3)$ & $1.12(3)$ & $8.73(-)$ & $(3-4)$ & - & $\mathrm{L} 3.5 \mathrm{~V}$ & $<0.5$ & $<0.5$ & $8.7^{\mathrm{a}}$ \\
\hline 2MASSW J0051107-154417 & $1.57(2)$ & $1.43(4-5)$ & $1.06(2-3)$ & $8.62(-)$ & (4) & - & $\mathrm{L} 3.5 \mathrm{~V}$ & $<2$ & 10 & 30 \\
\hline 2MASSW J0058425-065123 & $1.16(0)$ & $0.73(0)$ & $0.74(\mathrm{M} 9)$ & $6.53(-)$ & (0) & - & Lo V & 2 & $<1$ & 32 \\
\hline 2MASSI J0103320+193536 & $1.96(5-6)$ & $2.05(7)$ & $1.47(5)$ & $16.36(6)$ & - & - & L6 V & $<1$ & 12 & 29 \\
\hline 2MASSW J0135358+120522 & $1.39(1)$ & $1.01(1-2)$ & $0.93(1-2)$ & $5.76(-)$ & $(2)$ & - & $\mathrm{L} 1.5 \mathrm{~V}$ & 7 & $<0.5$ & 28 \\
\hline 2MASSW J0205034+125142 & $2.19(5)$ & $1.93(6-7)$ & $1.24(4)$ & $10.95(5)$ & $(4-5)$ & - & L5 V & $<1$ & $<1$ & 27 \\
\hline 2MASSW J0208183+254253 & $1.37(1)$ & $0.86(1)$ & $0.87(1)$ & $6.52(-)$ & (2) & - & L1 V & $<0.5$ & $<0.5$ & 25 \\
\hline 2MASSW J0208236+273740 & $2.14(5)$ & $1.41(4)$ & $1.57(6-7)$ & $10.66(5)$ & $(4-5)$ & - & L5 V & $<1$ & $<1$ & 29 \\
\hline 2MASSW J0208549+250048 & $1.89(4)$ & $1.51(4-5)$ & $1.72(8+)$ & $11.61(5)$ & $(5)$ & - & L5 $\mathrm{V}$ & $<1$ & 12 & 36 \\
\hline 2MASSI J0224367+253704 & $1.56(2)$ & $1.08(2)$ & $1.21(3-4)$ & $6.19(-)$ & $(2)$ & - & $\mathrm{L} 2 \mathrm{~V}$ & $<1$ & $<1$ & 66 \\
\hline 2MASSI J0302012+135814 & $1.81(4)$ & $1.13(3)$ & $1.11(3)$ & $6.68(-)$ & (2) & - & L3 $\mathrm{V}$ & $<2$ & 5 & 56 \\
\hline 2MASSW J0306268+154514 & $1.59(7)$ & - & - & $13.88(5)$ & - & $(6-8)$ & L6: V & $<6$ & $<6$ & 44 \\
\hline 2MASSW J0309088-194938 & $1.72(3)$ & - & - & $11.54(5)$ & (4) & $(5)$ & $\mathrm{L} 4.5 \mathrm{~V}$ & $<7$ & $<6$ & 34 \\
\hline 2MASSW J0310599+164816 & $1.24(8)$ & - & - & $31.55(8)$ & - & $(8+)$ & $\mathrm{L} 8 \mathrm{~V}$ & $<3$ & 5 & 20 \\
\hline 2MASSI J0328426+230205 & $1.16(8+)$ & - & - & $31.21(8)$ & - & $(8+)$ & $\mathrm{L} 8 \mathrm{~V}$ & $<4$ & $<3$ & 24 \\
\hline 2MASSW J0337036-175807 & $1.62(3)$ & - & - & $11.33(5)$ & $(4-5)$ & $(5)$ & $\mathrm{L} 4.5 \mathrm{~V}$ & $<5$ & 8 & 29 \\
\hline 2MASSI J0409095+210439 & $1.67(3)$ & $1.10(2-3)$ & $1.21(3-4)$ & $7.71(-)$ & (3) & - & L.3 V & $<1$ & $<2$ & 38 \\
\hline 2MASSW J0708213+295035 & $2.18(5)$ & - & - & $13.00(5)$ & (5) & $(5-6)$ & L5 $\mathrm{V}$ & $<2$ & $<5$ & 44 \\
\hline 2MASSW J0740096+321203 & $2.46(5)$ & $1.54(4-5)$ & $1.38(4-5)$ & $10.13(5)$ & (4) & - & $\mathrm{L} 4.5 \mathrm{~V}$ & $<2$ & $<2$ & 37 \\
\hline 2MASSI J0746425+200032 & $1.29(0-1)$ & $0.74(0-1)$ & $0.88(1)$ & $6.41(-)$ & $(0-1)$ & - & $\mathrm{L} 0.5 \mathrm{~V}$ & 2 & $<0.5$ & $12.3^{\mathrm{a}}$ \\
\hline 2MASSI J0753321+291711 & $1.50(2)$ & $1.02(2)$ & $1.16(3)$ & $6.33(-)$ & $(2)$ & - & $\mathrm{L} 2 \mathrm{~V}$ & $<0.5$ & $<1$ & 42 \\
\hline 2MASSI J0756252+124456 & $1.47(7-8)$ & - & - & $15.19(6)$ & - & $(5)$ & L6 V & $<3$ & 15 & 35 \\
\hline 2MASSW J0801405+462850 & $1.62(7)$ & $1.87(6)$ & $1.82(8+)$ & $13.03(5)$ & - & - & $\mathrm{L} 6.5 \mathrm{~V}$ & $<2$ & $<2$ & 28 \\
\hline 2MASSW J0820299+450031 & $2.38(5)$ & $1.63(5-6)$ & $1.54(6)$ & $11.76(5)$ & (4) & - & L5 V & $<2$ & $<4$ & 36 \\
\hline 2MASSI J0825196+211552 & $1.44(7-8)$ & $2.10(7)$ & $1.43(5)$ & $25.22(7-8)$ & - & - & $\mathrm{L} 7.5 \mathrm{~V}$ & $<2$ & 10 & 12 \\
\hline 2MASSW J0829066+145622 & $1.58(2)$ & $1.01(2)$ & $1.08(3)$ & $5.70(-)$ & (2) & - & L2 V & $<0.5$ & $<0.5$ & 30 \\
\hline 2MASSW J0829570+265510 & $2.13(5)$ & - & - & $17.72(6-7)$ & - & $(7)$ & $\mathrm{L} 6.5 \mathrm{~V}$ & $<10$ & 18 & 37 \\
\hline 2MASSW J0832045-012835 & $1.46(1-2)$ & $1.00(2)$ & $0.90(1-2)$ & $6.58(-)$ & $(2)$ & - & $\mathrm{L} 1.5 \mathrm{~V}$ & 2 & $<1$ & 25 \\
\hline 2MASSW J0920122+351742 & $1.68(6-7)$ & $1.84(6)$ & $1.72(8+)$ & $20.61(7)$ & - & - & $\mathrm{L} 6.5 \mathrm{~V}$ & $<0.5$ & $<0.5$ & 21 \\
\hline 2MASSW J0928397-160312 & $1.54(2)$ & $1.11(2-3)$ & $1.03(2)$ & $5.90(-)$ & (2) & - & L2 V & $<0.5$ & $<1$ & 39 \\
\hline 2MASSW J0929336+342952 & $1.22(8)$ & - & - & $22.39(7)$ & - & $(8)$ & $\mathrm{L} 8 \mathrm{~V}$ & $<3$ & $\leq 11$ & 22 \\
\hline 2MASSW J0944027+313132 & $1.58(2)$ & $1.07(2)$ & $0.89(1-2)$ & $6.65(-)$ & $(2)$ & - & L2 $\mathrm{V}$ & $\leq 1$ & $<1$ & 44 \\
\hline 2MASSW J0951054+355801 & $1.67(3)$ & - & - & $15.94(6)$ & - & $(6-7)$ & L6 V & $<5$ & $<10$ & 45 \\
\hline 2MASSI J1029216+162652 & $1.72(3-4)$ & $1.08(2)$ & $1.03(2)$ & $6.86(-)$ & $(2-3)$ & - & $\mathrm{L} 2.5 \mathrm{~V}$ & 0.5 & $<0.5$ & 23 \\
\hline 2MASSW J1035245+250745 & $1.41(1)$ & $0.88(1)$ & $0.87(1)$ & $6.15(-)$ & $(1-2)$ & - & L1 V & $<1$ & $<1$ & 35 \\
\hline
\end{tabular}


TABLE 2. (continued)

\begin{tabular}{|c|c|c|c|c|c|c|c|c|c|c|}
\hline $\begin{array}{l}\text { Name of L Dwarf } \\
\text { (1) }\end{array}$ & $\begin{array}{c}\mathrm{CrH}-\mathrm{a} \\
(2)\end{array}$ & $\begin{array}{c}\text { Rb-b/TiO-b } \\
\text { (3) }\end{array}$ & $\begin{array}{c}\mathrm{Cs}-\mathrm{a} / \mathrm{VO}-\mathrm{b} \\
(4)\end{array}$ & $\begin{array}{l}\text { Color-d } \\
\qquad(5)\end{array}$ & $\begin{array}{l}\text { K I Fit } \\
(6)\end{array}$ & $\begin{array}{l}\text { Oxide } \\
\text { Fit } \\
(7)\end{array}$ & $\begin{array}{l}\text { Spectral } \\
\text { Type } \\
(8)\end{array}$ & $\begin{array}{c}\mathrm{H} \alpha \\
\mathrm{EW}(\AA) \\
(9)\end{array}$ & $\begin{array}{c}\text { Li I } \\
\text { EW }(\AA) \\
(10)\end{array}$ & $\begin{array}{l}\text { Est. Dist } \\
\quad(p c) \\
(11)\end{array}$ \\
\hline 2MASSW J1102337-235945 & 1.35(1:) & - & - & $9.03(-)$ & $(4)$ & $(5)$ & $\mathrm{L} 4.5 \mathrm{~V}$ & $<5$ & $\leq 5$ & 53 \\
\hline 2MASSW J1112257+354813 & $1.98(5)$ & $1.60(5)$ & $1.28(4)$ & $9.90(-)$ & (4) & - & L4.5 V & $\leq 1$ & 9 & $21.7^{\mathrm{a}}$ \\
\hline 2MASSW J1123556+412228 & $1.55(2)$ & $1.16(3)$ & $1.03(2)$ & $5.91(-)$ & $(2)$ & - & $\mathrm{L} 2.5 \mathrm{~V}$ & $\overline{<} 1$ & $<1$ & 51 \\
\hline 2MASSW J1239272+551537 & $2.05(5)$ & $1.71(5-6)$ & $1.40(5)$ & $11.45(5)$ & $(4-5)$ & - & L5 $\mathrm{V}$ & $<3$ & 14 & 17 \\
\hline 2MASSW J1246467+402715 & $1.85(4)$ & $1.30(4)$ & $1.39(4-5)$ & $9.43(-)$ & (4) & - & $\mathrm{L} 4 \mathrm{~V}$ & $\leq 1$ & 11 & 25 \\
\hline 2MASSI J1332286+263508 & $1.49(2)$ & $1.06(2)$ & $1.02(2)$ & $5.92(-)$ & $(2)$ & - & $\mathrm{L} 2 \mathrm{~V}$ & $<2$ & $<2$ & 55 \\
\hline 2MASSW J1338261+414034 & $1.54(2)$ & $1.06(2)$ & $1.07(2-3)$ & $7.05(-)$ & (3) & - & $\mathrm{L} 2.5 \mathrm{~V}$ & $\leq 1$ & $<0.5$ & 23 \\
\hline 2MASSW J1343167+394508 & $2.36(5)$ & $1.77(6)$ & $1.48(5)$ & $13.03(5)$ & $(4-5)$ & - & L5 $\mathrm{V}$ & $<3$ & $\leq 20$ & 34 \\
\hline 2MASSW J1411175+393636 & $1.35(1)$ & $0.99(2)$ & $0.84(1)$ & $6.88(-)$ & $(2)$ & - & L1.5 V & $<1$ & $<1$ & 33 \\
\hline 2MASSW J1412244+163312 & $1.27(0-1)$ & $0.79(1)$ & $0.79(0-1)$ & $6.23(-)$ & $(0)$ & - & L0.5 V & 4 & $<0.5$ & 26 \\
\hline 2MASSW J1438549-130910 & $1.50(2)$ & - & - & $6.93(-)$ & $(2-3)$ & $(\sim 4)$ & L3: V & $<4$ & $<4$ & 38 \\
\hline 2MASSW J1449378+235537 & $1.18(0)$ & - & - & - & $(0)$ & $(0)$ & Lo V & - & $<10$ & 62 \\
\hline 2MASSW J1507476-162738 & $1.99(5)$ & $1.46(4-5)$ & $1.39(4-5)$ & $13.19(5)$ & $(5)$ & - & L5 $\mathrm{V}$ & $<0.5$ & $<0.5$ & $8:^{\mathrm{a}}$ \\
\hline 2MASSW J1523226+301456 & $1.19(8+)$ & - & - & $35.16(8+)$ & - & $(8)$ & $\mathrm{L} 8 \mathrm{~V}$ & $<15$ & 9 & $18.6^{\mathrm{a}}$ \\
\hline 2MASSI J1526140+204341 & $1.91(6)$ & $2.43(7-8)$ & $2.16(8+)$ & - & - & - & $\mathrm{L} 7 \mathrm{~V}$ & - & $<10$ & 19 \\
\hline 2MASSI J1600054+170832 & $1.57(2)$ & $0.72(0)$ & $0.91(1-2)$ & $6.94(-)$ & $(2)$ & - & L1.5 V & $<1.5$ & $<2$ & 62 \\
\hline 2MASSW J1615441+355900 & $1.86(4)$ & $1.02(2)$ & $1.15(3)$ & $7.68(-)$ & (3) & - & L3 V & $<1$ & $<1$ & 24 \\
\hline 2MASSI J1656188+283506 & $1.75(3-4)$ & - & - & $10.74(5)$ & (4) & $(5)$ & $\mathrm{L} 4.5 \mathrm{~V}$ & $<6$ & $<3$ & 56 \\
\hline 2MASSI J1711457+223204 & $1.96(5-6)$ & - & - & $16.87(6-7)$ & - & $(6-7)$ & $\mathrm{L} 6.5 \mathrm{~V}$ & $<4$ & $\leq 7$ & 36 \\
\hline 2MASSI J1726000+153819 & $1.44(1-2)$ & $1.31(4)$ & $0.93(1-2)$ & $8.44(-)$ & $(2)$ & - & $\mathrm{L} 2 \mathrm{~V}$ & $<2$ & 6 & 42 \\
\hline 2MASSW J1728114+394859 & $1.52(7)$ & - & - & $19.76(7)$ & - & (7) & $\mathrm{L} 7 \mathrm{~V}$ & $<7$ & $<4$ & 20 \\
\hline 2MASSW J1743415+212707 & $1.49(2)$ & $1.31(4)$ & $0.99(2)$ & $5.84(-)$ & $(3)$ & - & $\mathrm{L} 2.5 \mathrm{~V}$ & 6 & $<1$ & 42 \\
\hline 2MASSW J1841086+311727 & $1.41(1)$ & $1.06(2)$ & $1.10(3)$ & $5.04(-)$ & $(2 ?)$ & - & L4 $\mathrm{V}$ pec $^{\mathrm{b}}$ & $<2$ & $<2$ & 40 \\
\hline 2MASSI J2054358+151904 & $1.04(\mathrm{M} 9)$ & - & - & $5.38(-)$ & $(0)$ & $(2-3)$ & L1: V & $<6$ & $<4$ & 75 \\
\hline 2MASSI J2057153+171515 & $1.44(1-2)$ & $0.93(1-2)$ & $0.83(0-1)$ & $5.61(-)$ & (2) & - & L1.5 V & $<2$ & $<1$ & 61 \\
\hline 2MASSW J2101154+175658 & $2.09(5-6)$ & - & - & $27.61(7-8)$ & - & $(7-8)$ & $\mathrm{L} 7.5 \mathrm{~V}$ & $<7$ & $<5$ & 29 \\
\hline 2MASSW J2206540-421721 & $1.26(0-1)$ & $1.39(4)$ & $1.03(2)$ & $6.75(-)$ & (2) & - & $\mathrm{L} 2 \mathrm{~V}$ & $\leq 2$ & 8 & 41 \\
\hline 2MASSW J2208136+292121 & $1.32(1)$ & $0.98(2)$ & $1.06(2-3)$ & $6.73(-)$ & $(0$ pec $)$ & - & L2 $\mathrm{V}$ pec $^{\mathrm{c}}$ & $<2$ & 4 & 48 \\
\hline 2MASSW J2224438-015852 & $1.99(5)$ & $1.56(4-5)$ & $1.20(3-4)$ & $10.71(5)$ & $(4-5)$ & - & $\mathrm{L} 4.5 \mathrm{~V}$ & 1 & $<0.5$ & 14 \\
\hline
\end{tabular}

${ }^{\text {a }}$ Distances determined via trigonometric parallax. See text and Table 3 for details.

${ }^{\mathrm{b}}$ This object has a peculiar spectrum showing feature strengths that match those of an L4 dwarf although the overall color between 6300 and $10000 \AA$ is too blue to be even early L.

${ }^{c}$ This object has a peculiar spectrum showing narrow K I lines like that of an LO dwarf but TiO bands with strengths simliar to an L4 dwarf. 
TABle 3. Late-M, L, and T Dwarfs with Measured Trigonometric Parallaxes

\begin{tabular}{|c|c|c|c|c|}
\hline $\begin{array}{c}\text { Name of Dwarf } \\
\text { (1) }\end{array}$ & $\begin{array}{l}\text { Type } \\
(2)\end{array}$ & $\begin{array}{l}\text { Ref. for } \pi_{t r i g} \\
\text { (3) }\end{array}$ & $\begin{array}{c}M_{J}(\mathrm{mag}) \\
\quad(4)\end{array}$ & $\begin{array}{c}M_{K s}(\mathrm{mag}) \\
\quad(5)\end{array}$ \\
\hline BRI 1222-1222 & M9 V & Tinney (1996) & $11.35 \pm 0.15$ & $10.16 \pm 0.15$ \\
\hline TVLM 868-110639 & M9 V & Tinney (1993) & $11.43 \pm 0.08$ & $10.17 \pm 0.08$ \\
\hline LHS 2065 & M9 V & Monet et al. (1992) & $11.58 \pm 0.04$ & $10.30 \pm 0.04$ \\
\hline LHS 2924 & M9 V & Monet et al. (1992) & $11.78 \pm 0.04$ & $10.55 \pm 0.04$ \\
\hline LP 944-20 & M9 V & Tinney (1996) & $12.24 \pm 0.05$ & $11.04 \pm 0.05$ \\
\hline BRI 0021-0214 & M9.5 V & Tinney (1993) & $11.60 \pm 0.09$ & $10.12 \pm 0.09$ \\
\hline 2MASSW J0149090+295613 & M9.5 V & Dahn (priv. comm.) & $11.68 \pm 0.05$ & $10.26 \pm 0.05$ \\
\hline 2MASP J0345432+254023 & L0 V & Dahn (priv. comm.) & $11.88 \pm 0.05^{\mathrm{a}}$ & $10.55 \pm 0.06^{\mathrm{a}}$ \\
\hline 2MASSI J0746425+200032 & $\mathrm{L} 0.5 \mathrm{~V}$ & Dahn (priv. comm.) & $11.30 \pm 0.04$ & $10.05 \pm 0.04$ \\
\hline 2MASSW J1439284+192915 & L1 V & Dahn (priv. comm.) & $11.98 \pm 0.04$ & $10.80 \pm 0.04$ \\
\hline Kelu-1 & $\mathrm{L} 2 \mathrm{~V}$ & ahn (priv. comm.) & $11.96 \pm 0.09$ & $10.39 \pm 0.09$ \\
\hline 2MASSW J1146345+223053 & L3 V & Dahn (priv. comm.) & $12.89 \pm 0.08^{\mathrm{b}}$ & $11.29 \pm 0.08^{\mathrm{b}}$ \\
\hline DENIS-P J1058.7-1548 & L3 V & hhn (priv. comm.) & $2.96 \pm 0.05$ & $11.31 \pm 0.05$ \\
\hline 2MASSW J0036159+182110 & L3.5 V & ahn (priv. comm.) & $12.74 \pm 0.05$ & $11.33 \pm 0.04$ \\
\hline 2MASSW J0326137+29 & L3.5 V & ahn (priv. comm.) & $12.84 \pm 0.12$ & $11.24 \pm 0.12$ \\
\hline 2MASSW J1112257+354813 & $\mathrm{L} 4.5 \mathrm{~V}$ & erryman et al. (1997) & $12.89 \pm 0.06^{\mathrm{d}}$ & $11.01 \pm 0.07^{\mathrm{d}}$ \\
\hline 2MASSW J1328550+211449 & L5 V & Dahn (priv. comm.) & $13.14 \pm 0.42$ & $11.35 \pm 0.42$ \\
\hline GJ 1001B & L5 V & van Altena et al. (1995) & $13.20 \pm 0.04^{\mathrm{d}}$ & $11.50 \pm 0.04^{\mathrm{d}}$ \\
\hline 2MASSW J1507476-162738 & L5 V & Dahn (priv. comm.) & $13.41 \pm 0.39$ & $11.89 \pm 0.39$ \\
\hline DENIS-P J1228.2-1547 & L5 V & Dahn (priv. comm.) & $13.67 \pm 0.13^{\mathrm{b}}$ & $12.10 \pm 0.13^{\mathrm{b}}$ \\
\hline 2MASSs J0850359+105716 & L6 $\mathrm{V}$ & Dahn (priv. comm.) & $14.02 \pm 0.36$ & $12.03 \pm 0.35$ \\
\hline DENIS-P J0205.4-1159 & L7 V & & $14.09 \pm 0.09^{\mathrm{b}}$ & $12.53 \pm 0.09^{\mathrm{b}}$ \\
\hline 2MASSW J1632291+190441 & L8 V & Dahn (priv. comm.) & $14.73 \pm 0.13$ & $12.85 \pm 0.12$ \\
\hline 2MASSW J1523226+301456 & $\mathrm{L} 8 \mathrm{~V}$ & Perryman et al. (1997) & $14.98 \pm 0.12^{\mathrm{d}}$ & $12.89 \pm 0.09^{\mathrm{d}}$ \\
\hline Gl 229B & $\mathrm{T}$ dwarf & Perryman et al. (1997) & $15.4 \pm 0.1^{\mathrm{d}}$ & $15.5 \pm 0.1^{\mathrm{d}}$ \\
\hline Gl 570D & $\mathrm{T}$ dwarf & Perryman et al. (1997) & $16.47 \pm 0.05^{\mathrm{d}}$ & $16.41 \pm 0.17^{\mathrm{d}}$ \\
\hline
\end{tabular}

\section{Notes to Table 3 .}

All spectral types are on the system of Paper I for the L and T dwarfs and Kirkpatrick et al. 1995 for the late-M dwarfs. Photometry is taken from 2MASS except for Gl 229B, where photometry is taken from Matthews et al. (1996). Absolute magnitudes derived from USNO data (listed as "Dahn, priv. comm.") use parallaxes updated on January 4, 2000.

${ }^{a}$ Absolute magnitudes are not corrected for the possible duplicity noted in Reid et al. (1999).

${ }^{\mathrm{b}}$ Absolute magnitudes have been dimmed by $0.75 \mathrm{mag}$ to account for the fact that this system is a close, equal-magnitude double (Koerner et al. 1999; see also Martín et al. 1999a for DENIS-P J1228.2-1547.).

${ }^{\mathrm{c}}$ Also known as Gl 417B, the widely separated companion to Gl 417A (Kirkpatrick et al. 2000).

${ }^{\mathrm{d}}$ The measured parallax for the primary is assumed here for the companion.

${ }^{\mathrm{e}}$ Also known as Gl 584C, the widely separated companion to Gl 584AB (Kirkpatrick et al. 2000). 
TABle 4. L and T Dwarfs within (or Possibly within) 25 Parsecs of the Sun

\begin{tabular}{|c|c|c|c|c|c|c|c|}
\hline \multirow[b]{2}{*}{$\begin{array}{l}\text { Name of Dwarf } \\
\text { (1) }\end{array}$} & \multirow[b]{2}{*}{$\begin{array}{c}\text { Discovery Paper } \\
(2)\end{array}$} & \multirow[b]{2}{*}{$\begin{array}{c}\text { Type }^{\mathrm{a}} \\
(3)\end{array}$} & \multirow[b]{2}{*}{$\begin{array}{c}J(\mathrm{mag})^{\mathrm{b}} \\
(4)\end{array}$} & \multirow[b]{2}{*}{$\begin{array}{c}K_{s}(\mathrm{mag})^{\mathrm{b}} \\
(5)\end{array}$} & \multicolumn{3}{|c|}{ Distance $(\mathrm{pc})^{\mathrm{c}}$} \\
\hline & & & & & $\begin{array}{c}\text { from } \\
J \\
(6)\end{array}$ & $\begin{array}{c}\text { from } \\
K_{s} \\
(7)\end{array}$ & $\begin{array}{c}\text { from } \\
\pi_{\text {trig }} \\
(8)\end{array}$ \\
\hline 2MASSI J0746425+200032 & Reid et al. (2000) & L0.5 V & $11.74 \pm 0.03$ & $10.49 \pm 0.03$ & $\cdots$ & $\cdots$ & 12.3 \\
\hline 2MASSW J1412244+163312 & this paper & $\mathrm{L} 0.5 \mathrm{~V}$ & $13.89 \pm 0.04$ & $12.59 \pm 0.03$ & 25 & 26 & $\cdots$ \\
\hline 2MASSW J1439284+192915 & Paper I & L1 V & $12.76 \pm 0.04$ & $11.58 \pm 0.04$ & $\ldots$ & $\ldots$ & 14.3 \\
\hline 2MASSW J1300425+191235 & Gizis et al. (2000) & L1 V & $12.71 \pm 0.02$ & $11.61 \pm 0.03$ & 14 & 16 & $\ldots$ \\
\hline 2MASSW J1108307+683017 & Gizis et al. (2000) & L1 V & $13.14 \pm 0.03$ & $11.60 \pm 0.03$ & 17 & 16 & $\ldots$ \\
\hline 2MASSW J1658037+702701 & Gizis et al. (2000) & L1 V & $13.31 \pm 0.03$ & $11.92 \pm 0.03$ & 18 & 18 & $\ldots$ \\
\hline 2MASSW J0208183+254253 & this paper & L1 V & $14.02 \pm 0.03$ & $12.58 \pm 0.04$ & 25 & 25 & $\ldots$ \\
\hline 2MASSW J0832045-012835 & this paper & $\mathrm{L} 1.5 \mathrm{~V}$ & $14.13 \pm 0.03$ & $12.69 \pm 0.03$ & 25 & 25 & $\cdots$ \\
\hline DENIS-P J1441-0945 & Martín et al. (1999b) & $\sim \mathrm{L} 1 \mathrm{~V}$ & $14.00 \pm 0.03$ & $12.64 \pm 0.04$ & 25 & 26 & $\cdots$ \\
\hline Kelu-1 & Ruiz et al. (1997) & $\mathrm{L} 2 \mathrm{~V}$ & $13.38 \pm 0.03$ & $11.81 \pm 0.03$ & $\cdots$ & $\ldots$ & 19.2 \\
\hline 2MASSW J0015447+351603 & this paper & $\mathrm{L} 2 \mathrm{~V}$ & $13.82 \pm 0.04$ & $12.24 \pm 0.03$ & 20 & 19 & $\ldots$ \\
\hline 2MASSW J1338261+414034 & this paper & $\mathrm{L} 2.5 \mathrm{~V}$ & $14.22 \pm 0.03$ & $12.75 \pm 0.04$ & 23 & 23 & $\ldots$ \\
\hline 2MASSI J1029216+162652 & this paper & $\mathrm{L} 2.5 \mathrm{~V}$ & $14.31 \pm 0.04$ & $12.61 \pm 0.04$ & 24 & 22 & $\ldots$ \\
\hline G $196-3 \mathrm{~B}$ & Rebolo et al. (1998) & $\mathrm{L} 2 \mathrm{~V}$ & $14.90 \pm 0.05$ & $12.81 \pm 0.13$ & 34 & 25 & $\cdots$ \\
\hline 2MASSW J0036159+182110 & Reid et al. (2000) & L3.5 V & $12.44 \pm 0.04$ & $11.03 \pm 0.03$ & $\cdots$ & $\cdots$ & 8.7 \\
\hline 2MASSW J1506544+132106 & Gizis et al. (2000) & L3 V & $13.41 \pm 0.03$ & $11.75 \pm 0.03$ & 15 & 14 & $\cdots$ \\
\hline DENIS-P J1058.7-1548 & Delfosse et al. (1997) & L3 V & $14.18 \pm 0.03$ & $12.53 \pm 0.03$ & $\cdots$ & $\cdots$ & 17.5 \\
\hline SDSSp J120358.19+001550.3 & Fan et al. (2000) & $\sim \mathrm{L} 3 \mathrm{~V}$ & $14.02 \pm 0.03$ & $12.48 \pm 0.03$ & 19 & 19 & $\cdots$ \\
\hline DENIS-P J1047-1815 & Martín et al. (1999b) & $\sim \mathrm{L} 3 \mathrm{~V}$ & $14.20 \pm 0.03$ & $12.90 \pm 0.03$ & 21 & 24 & $\cdots$ \\
\hline 2MASSW J1615441+355900 & this paper & L3 V & $14.55 \pm 0.04$ & $12.89 \pm 0.05$ & 25 & 23 & $\cdots$ \\
\hline 2MASSW J2224438-015852 & this paper & L4.5 V & $14.05 \pm 0.03$ & $12.02 \pm 0.03$ & 15 & 13 & $\cdots$ \\
\hline 2MASSW J1112257+354813 & this paper & $\mathrm{L} 4.5 \mathrm{~V}$ & $14.57 \pm 0.04$ & $12.69 \pm 0.05$ & $\cdots$ & $\ldots$ & 21.7 \\
\hline 2MASSW J1246467+402715 & this paper & $\mathrm{L} 4 \mathrm{~V}$ & $15.00 \pm 0.04$ & $13.30 \pm 0.04$ & 26 & 25 & $\cdots$ \\
\hline 2MASSW J1507476-162738 & Reid et al. (2000) & L5 V & $12.82 \pm 0.03$ & $11.30 \pm 0.03$ & $\cdots$ & $\cdots$ & 8.: \\
\hline GJ 1001B & Goldman et al. (1999) & L5 V & $13.10 \pm 0.03$ & $11.40 \pm 0.03$ & $\ldots$ & $\ldots$ & 9.6 \\
\hline SDSSp J053951.99-005902.0 & Fan et al. (2000) & $\sim \mathrm{L} 5 \mathrm{~V}$ & $13.99 \pm 0.03$ & $12.58 \pm 0.03$ & 13 & 15 & $\cdots$ \\
\hline 2MASSW J1239272+551537 & this paper & L5 V & $14.67 \pm 0.03$ & $12.74 \pm 0.03$ & 18 & 16 & $\cdots$ \\
\hline DENIS-P J1228.2-1547AB & Delfosse et al. (1997) & $\mathrm{L} 5 \mathrm{~V}^{\mathrm{e}}$ & $14.38 \pm 0.04^{\mathrm{e}}$ & $12.81 \pm 0.03^{\mathrm{e}}$ & $\cdots$ & $\cdots$ & 19.6 \\
\hline 2MASSW J0205034+125142 & this paper & L5 V & $15.69 \pm 0.06$ & $13.68 \pm 0.05$ & 29 & 25 & $\cdots$ \\
\hline 2MASSW J0920122+351742 & this paper & $\mathrm{L} 6.5 \mathrm{~V}$ & $15.59 \pm 0.07$ & $13.93 \pm 0.08$ & 20 & 22 & $\cdots$ \\
\hline 2MASSI J0825196+211552 & this paper & L7.5 V & $15.12 \pm 0.04$ & $13.05 \pm 0.04$ & 13 & 12 & $\cdots$ \\
\hline DENIS-P J0205.4-1159AB & Delfosse et al. (1997) & $\mathrm{L} 7 \mathrm{~V}^{\mathrm{e}}$ & $14.55 \pm 0.03^{\mathrm{e}}$ & $12.99 \pm 0.04^{\mathrm{e}}$ & .. & $\ldots$ & 17.5 \\
\hline 2MASSI J1526140+204341 & this paper & L7 V & $15.62 \pm 0.07$ & $13.92 \pm 0.06$ & 18 & 20 & $\ldots$ \\
\hline 2MASSW J1728114+394859 & this paper & $\mathrm{L} 7 \mathrm{~V}$ & $15.96 \pm 0.08$ & $13.90 \pm 0.05$ & 22 & 19 & $\ldots$ \\
\hline 2MASSW J0030300-145033 & this paper & $\mathrm{L} 7 \mathrm{~V}$ & $16.79 \pm 0.16$ & $14.38 \pm 0.08$ & 32 & 24 & $\cdots$ \\
\hline DENIS-P J0255-4700 & Martín et al. (1999b) & $\sim \mathrm{L} 8 \mathrm{~V}$ & $13.23 \pm 0.03$ & $11.53 \pm 0.03$ & 5 & 5 & $\ldots$ \\
\hline 2MASSW J1632291+190441 & Paper I & L8 V & $15.86 \pm 0.07$ & $13.98 \pm 0.05$ & $\cdots$ & $\cdots$ & 16.8 \\
\hline SDSSp J132629.82-003831.5 & Fan et al. (2000) & $\widetilde{\mathrm{L} 8 \mathrm{~V}}$ & $16.11 \pm 0.07$ & $14.23 \pm 0.07$ & 18 & 18 & $\cdots$ \\
\hline 2MASSW J1523226+301456 ${ }^{\mathrm{f}}$ & this paper & $\mathrm{L} 8 \mathrm{~V}$ & $16.32 \pm 0.11$ & $14.24 \pm 0.07$ & $\cdots$ & $\cdots$ & 18.6 \\
\hline 2MASSW J0310599+164816 & this paper & L8 V & $16.43 \pm 0.11$ & $14.40 \pm 0.10$ & 21 & 20 & $\ldots$ \\
\hline
\end{tabular}


TABLE 4. (continued)

\begin{tabular}{|c|c|c|c|c|c|c|c|}
\hline \multirow[b]{2}{*}{$\begin{array}{c}\text { Name of Dwarf } \\
\text { (1) }\end{array}$} & \multirow[b]{2}{*}{$\begin{array}{c}\text { Discovery Paper } \\
(2)\end{array}$} & \multirow[b]{2}{*}{$\begin{array}{l}\text { Type }^{\mathrm{a}} \\
(3)\end{array}$} & \multirow[b]{2}{*}{$\begin{array}{c}J(\operatorname{mag})^{b} \\
(4)\end{array}$} & \multirow[b]{2}{*}{$\begin{array}{c}K_{s}(\operatorname{mag})^{\mathrm{b}} \\
(5)\end{array}$} & \multicolumn{3}{|c|}{ Distance $(\mathrm{pc})^{\mathrm{c}}$} \\
\hline & & & & & $\begin{array}{c}\text { from } \\
\quad J \\
(6)\end{array}$ & $\begin{array}{c}\text { from } \\
K_{s} \\
(7)\end{array}$ & $\begin{array}{c}\text { from } \\
\pi_{\text {trig }} \\
(8)\end{array}$ \\
\hline 2MASSW J0929336+342952 & this paper & L8 V & $16.60 \pm 0.13$ & $14.62 \pm 0.11$ & 23 & 22 & $\cdots$ \\
\hline 2MASSW J0328426+230205 & this paper & L8 V & $16.67 \pm 0.14$ & $14.84 \pm 0.13$ & 23 & 24 & $\cdots$ \\
\hline Gl 229B & Nakajima et al. (1995) & $\mathrm{T}$ dwarf & $14.4 \pm 0.1^{\mathrm{g}}$ & $14.5 \pm 0.1^{\mathrm{g}}$ & $\cdots$ & $\cdots$ & 5.8 \\
\hline Gl 570D & Burgasser et al. (2000a) & $\mathrm{T}$ dwarf & $15.33 \pm 0.05$ & $15.27 \pm 0.17$ & $\cdots$ & $\cdots$ & 5.9 \\
\hline 2MASSW J0559191-140448 & Burgasser et al. (2000c) & $\mathrm{T}$ dwarf & $13.83 \pm 0.03$ & $13.61 \pm 0.05$ & 6 & $\cdots$ & $\cdots$ \\
\hline 2MASSW J1225543-273947 & Burgasser et al. (1999) & $\mathrm{T}$ dwarf & $15.23 \pm 0.05$ & $15.06 \pm 0.15$ & 10 & $\cdots$ & $\cdots$ \\
\hline SDSSp J162414.37+002915.6 & Strauss et al. (1999) & $\mathrm{T}$ dwarf & $15.53 \pm 0.03^{\mathrm{h}}$ & $15.70 \pm 0.05^{\mathrm{h}}$ & 12 & $\cdots$ & $\cdots$ \\
\hline SDSSp J134646.45-003150.4 & Tsvetanov et al. (2000) & $\mathrm{T}$ dwarf & $15.82 \pm 0.05^{\mathrm{h}}$ & $15.84 \pm 0.07^{\mathrm{h}}$ & 13 & $\cdots$ & $\cdots$ \\
\hline 2MASSI J1047539+212423 & Burgasser et al. (1999) & $\mathrm{T}$ dwarf & $15.82 \pm 0.06$ & $>16.29$ & 13 & $\cdots$ & $\cdots$ \\
\hline 2MASSW J1217111-031113 & Burgasser et al. (1999) & $\mathrm{T}$ dwarf & $15.85 \pm 0.07$ & $>15.91$ & 14 & $\cdots$ & $\cdots$ \\
\hline 2MASSW J1237392+652615 & Burgasser et al. (1999) & $\mathrm{T}$ dwarf & $15.90 \pm 0.06$ & $>15.90$ & 14 & $\cdots$ & \\
\hline
\end{tabular}

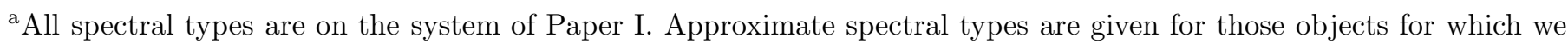
do not have spectra. For those objects, types have be (re-)assigned by eye based on the published spectra.

${ }^{\mathrm{b}}$ Photometry is from 2MASS unless otherwise noted.

${ }^{\mathrm{c}}$ Distances are computed from $\pi_{\text {trig }}$ when available. For $\mathrm{L}$ dwarfs without $\pi_{\text {trig }}$ measures, one distance estimate has been computed from the spectral type using equation (1) and the apparent $J$ magnitude, and another has been computed using equation (2) and the apparent $K_{s}$ magnitude. For $\mathrm{T}$ dwarfs without $\pi_{\text {trig }}$ measures, the method of Burgasser et al. 1999 has been used to estimate distances from the apparent $J$ magnitude, except for 2MASSW J0559-1404 whose distance estimate comes from Burgasser et al. 2000c.

${ }^{\mathrm{d}}$ Also known as Gl 417B. See Kirkpatrick et al. 2000.

e This is a combine measure for the $\mathrm{AB}$ pair.

${ }^{\mathrm{f}}$ Also known as Gl 584C. See Kirkpatrick et al. 2000.

'Photometry from Matthews et al. 1996.

${ }^{\mathrm{h}}$ Magnitude is from the discovery paper cited. Listed magnitude is $K$, not $K_{s}$. 
TABLE 5. Average Near-infrared Colors for Late-M and L Dwarfs ${ }^{\mathrm{a}}$

\begin{tabular}{|c|c|c|c|c|c|c|c|}
\hline $\begin{array}{l}\text { Sp. Type } \\
\text { (1) }\end{array}$ & $\begin{array}{c}<J-K_{s}> \\
(2)\end{array}$ & $\begin{array}{c}\sigma_{<J-K s>} \\
(3)\end{array}$ & $\begin{array}{c}<J-H> \\
(4)\end{array}$ & $\begin{array}{c}\sigma_{<J-H>} \\
(5)\end{array}$ & $\begin{array}{c}\left\langle H-K_{s}>\right. \\
(6)\end{array}$ & $\begin{array}{c}\sigma_{<H-K s>} \\
(7)\end{array}$ & $\begin{array}{l}\text { No. used in Ave. } \\
\text { (8) }\end{array}$ \\
\hline M8 & 1.09 & 0.05 & 0.68 & 0.03 & 0.41 & 0.03 & 14 \\
\hline M8.5 & 1.11 & 0.07 & 0.68 & 0.04 & 0.43 & 0.06 & 7 \\
\hline M9 & 1.17 & 0.03 & 0.72 & 0.03 & 0.46 & 0.02 & 9 \\
\hline M9.5 & 1.33 & 0.08 & 0.81 & 0.05 & 0.52 & 0.03 & 4 \\
\hline L0 & 1.40 & 0.07 & 0.82 & 0.08 & 0.58 & 0.13 & 3 \\
\hline L0.5 & 1.29 & 0.04 & 0.80 & 0.05 & 0.49 & 0.02 & 3 \\
\hline L1 & 1.43 & 0.21 & 0.80 & 0.11 & 0.63 & 0.16 & 8 \\
\hline L1.5 & 1.48 & 0.07 & 0.86 & 0.10 & 0.61 & 0.11 & 8 \\
\hline L2 & 1.71 & 0.17 & 1.02 & 0.09 & 0.70 & 0.09 & 12 \\
\hline L2.5 & 1.56 & 0.14 & 0.95 & 0.04 & 0.61 & 0.15 & 5 \\
\hline L3 & 1.72 & 0.14 & 1.03 & 0.05 & 0.68 & 0.10 & 9 \\
\hline L3.5 & 1.61 & 0.20 & 1.00 & 0.12 & 0.61 & 0.10 & 3 \\
\hline L4 & 1.87 & 0.16 & 1.18 & 0.18 & 0.68 & 0.08 & 4 \\
\hline L4.5 & 1.99 & 0.16 & 1.23 & 0.11 & 0.77 & 0.12 & 8 \\
\hline L5 & 1.87 & 0.20 & 1.17 & 0.12 & 0.70 & 0.10 & 10 \\
\hline L5.5 & 2.00 & 0.21 & 1.34 & 0.22 & 0.66 & 0.19 & 1 \\
\hline L6 & 2.06 & 0.12 & 1.18 & 0.29 & 0.88 & 0.19 & 5 \\
\hline L6.5 & 2.02 & 0.37 & 1.11 & 0.26 & 0.91 & 0.14 & 4 \\
\hline L7 & 1.94 & 0.37 & 1.18 & 0.19 & 0.76 & 0.20 & 4 \\
\hline L7.5 & 1.95 & 0.17 & 1.25 & 0.11 & 0.70 & 0.06 & 2 \\
\hline L8 & 1.96 & 0.11 & 1.24 & 0.18 & 0.72 & 0.14 & 5 \\
\hline
\end{tabular}

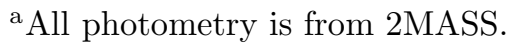

